\title{
On Families of Equi-Uniformly Distributed Sequences in Compact Spaces
}

\author{
By \\ P. C. BAAYEN in Amsterdam and G. Hrormberg in Eindhoven
}

\section{Introduction and preliminaries}

Let $X$ be a compact Hausdorff space, let $\mu$ be a regular normed Borel measure on $X$, and let $C(X)$ be the Banach space (under uniform norm $\left.\|f\|=\sup _{x \in X}|f(x)|\right)$ of all complex-valued continuous functions $f$ on $X$. Identifying measure and corresponding integral on $C(X)$ we shall use the notation $\mu(f)$ $=\int_{X} f(x) d \mu(x)$. A family $\mathcal{S}=\left\{\left(x_{\sigma, n}\right)_{n=1}^{\infty}: \sigma \in S\right\}$ of sequences in $X$ is called a family of equi- $\mu$-uniformly distributed sequences (in German: ,gleichmäßig $\mu$ gleichverteilt") if for every $f \in C(X)$ and for every real number $\varepsilon>0$ there exists an integer $N(f, \varepsilon)$, indepedent of $\sigma$, such that

$$
\left|\frac{1}{N} \sum_{n=1}^{N} f\left(x_{\sigma, n}\right)-\mu(f)\right| \leqq \varepsilon \text { for all } N \geqq N(f, \varepsilon)
$$

and for all $\sigma \in S$ (HLAWKa [10]).

The question arises as to the possible size of such a family $\widehat{S}$, both from the topological and from the measure-theoretic point of view (section 4). This question may be modified by restricting attention to sequences of a special type only, such as the tail sequences of a given $\mu$-uniformly distributed sequence (section 5,6 ) or the sequences formed by the successive powers of a generator in a compact monothetio group (section 2,3 ). In all of these modifications, a common feature of the answers to the above mentioned questions is the following: such a family $\mathcal{E}$ will in general be in some (topological) sense nowhere dense, it may be enlarged to one which is in some (topological) sense closed, and if the set of all sequences in question in some natural sense is given the measure 1 , then 5 may have measure abitrary close to 1 .

Before taking up the subject in detail we mention two facts which we shall have to use. First, in order to show that the sequences of a certain family 5 are equi- $\mu$-uniformly distributed it obviously suffices to establish (1) for the functions $f$ of a fixed set $F C C(X)$ having the property that finite complex linear combinations of elements of $F$ are uniformly dense in $C(X)([10] \S 2$, [12] Satz 4). This fact will in the following be refered to as Weyl's criterion.

Secondly we note that in the definition given above, $C(X)$ may be replaced by the set of all real-valued Borel measurable functions $f$ on $X$ having the 
following property: for every $\eta>0$ there exist real-valued continuous functions $f_{1}, f_{2}$ such that $f_{1} \leqq f \leqq f_{2}$ and $\mu\left(f_{2}-f_{1}\right) \leqq \eta$. Indeed, we then have

$$
\begin{aligned}
\frac{1}{N} \sum_{n=1}^{N} f_{1}\left(x_{\sigma, n}\right) & -\mu\left(f_{1}\right)-\eta \leqq \frac{1}{N} \sum_{n=1}^{N} f_{1}\left(x_{\sigma, n}\right)-\mu\left(f_{2}\right) \leqq \frac{1}{N} \sum_{n=1}^{N} f\left(x_{\sigma, n}\right)-\mu(f) \leqq \\
& \leqq \frac{1}{N} \sum_{n=1}^{X} f_{2}\left(x_{\sigma, n}\right)-\mu\left(f_{1}\right) \leqq \frac{1}{N} \sum_{n=1}^{N} f_{2}\left(x_{\sigma, n}\right)-\mu\left(f_{2}\right)+\eta
\end{aligned}
$$

and therefore

$$
\begin{aligned}
& \left|\frac{1}{N} \sum_{n=1}^{N} f\left(x_{\sigma, n}\right)-\mu(f)\right| \leqq \\
& \quad \leqq \max \left\{\left|\frac{1}{N} \sum_{n=1}^{N} f_{1}\left(x_{\sigma, n}\right)-\mu\left(f_{1}\right)\right|,\left|\frac{1}{N} \sum_{n=1}^{N} f_{2}\left(x_{\sigma, n}\right)-\mu\left(f_{2}\right)\right|\right\}+\eta .
\end{aligned}
$$

Since $\eta$ is arbitrary it is indeed possible to choose $N(f, \varepsilon)$ so large that for all $N \geqq N(f, \varepsilon)$ the right hand member is smaller than a given $\varepsilon>0$, uniformly in $\sigma$.

As a consequence, if $\mathfrak{S}=\left\{\left(x_{\sigma, n}\right)_{n=1}^{\infty}: \sigma \in S\right\}$ is a family of equi- $\mu$-uniformly distributed sequences, (1) will hold for any complex-valued (Borel measurable) function $f$ whose discontinuities are contained in a closed $\mu$-zero set, in particular for every characteristic function $\chi_{A}$ of a subset $A$ of $X$ whose boundary has $\mu$-measure zero and for the product of $\chi_{A}$ with any function $f \in C(X)$ (cf. [12] Satz 6, [7] I.).

As a case of particular interest, we already mentioned the set of sequences $\left(a^{n}\right)_{n=1}^{\infty}$ if $X$ is a compact monothetic group and if $a$ runs through the generators of $X$. Here, because of the one-to-one correspondence between generators and sequences, the answers to the questions referred to above may be given by statements about sets of generators of $X$. Since it is both instructive and convenient for our later considerations, we shall first deal with the special case of uniform distribution mod 1 before passing on to more general situations.

Some of the results of sections 2 and 3 have been announced by the authors in a talk given in October 1963 in the colloquium on uniform distribution at the Mathematical Center in Amsterdam.

\section{Uniform distribution mod 1}

Let $X$ be the additive group of reals mod 1 and let $\mu$ be ordinary Lebesgue measure on $\left[0,1\left[\right.\right.$. It is well known that the sequence $(n a)_{n=1}^{\infty}$ is $(\mu-)$ uniformly distributed $\bmod 1$ iff $a$ is irrational. Taking in Weyl's criterion for $F$ the set of functions $f_{k}(x)=e^{2 \pi i k x}$ ( $k$ an integer) we find that necessary and sufficient for the sequences $\left(x_{\sigma, n}\right)_{n=1}^{\infty}(\sigma \in S)$ to be equi-uniformly distributed mod $l$ is the existence, for every integer $k \geqq 1$ and for every real number $\varepsilon>0$, of an integer $N(k, \varepsilon)$, independent of $\sigma$, such that

$$
\left|\frac{1}{N} \sum_{n=1}^{N} e^{2 \pi i k x_{\sigma, n}}\right| \leqq \varepsilon \text { for all } N \geqq N(k, \varepsilon)
$$


and for all $\sigma \in S$. In what follows we shall denote by $[a]$ the greatest integer in $a$ and by $\hat{a}=a-[a]$ the residue of $a \bmod 1$. This should not cause confusion with the further use of brackets when replacing parentheses or signalling open or closed intervals.

Theorem 1. Let $A$ be a set of irrational numbers such that the sequences $(n a)_{n=1}^{\infty}(a \in A)$ are equi-uniformly distributed $\bmod 1$. Then the set of residues $\bmod 1 \hat{A}=\{\hat{a}: a \in A\}$ is nowhere dense in $[0,1[$.

Proof. Assume the contrary. Then we can find an integer $k \geqq 1$ such that the set $k A$ is dense in $[0,1[(\bmod 1)$. Given any integer $N \geqq 1$ and any real number $\delta(0<\delta<1)$ we can therefore find a number $a \in A$ such that $k a \in\left[0, \frac{\delta}{N}[\right.$ $(\bmod 1)$ and, a fortiori, $n k a \in[0, \delta[(\bmod 1)$ for $1 \leqq n \leqq N$. Thus, for these $n$, if $\delta$ has been chosen small enough, $e^{2 \pi i n k a}$ is close to 1 and so is $\left|\frac{1}{N} \sum_{n=1}^{N} e^{2 \pi i n k a}\right|$, in
contradiction to (3).

Corollary 1.1. Let a be irrational. Then the sequences $(n m a)_{n=1}^{\infty}(m=1,2, \ldots)$ are not equi-uniformly distributed $\bmod 1$.

Corollary 1.2. The family of all sequences $(n a)_{n=1}^{\infty}$ (a irrational) is not the union of countably many families of mod 1 equi.uniformly distributed sequences.

Corollary 1.3. Let $A$ and $\hat{A}$ be as in theorem 1. Then $\hat{A}$ has outer Lebesgue measure smaller than 1.

Theorem 2. Let $\delta(0<\delta<1)$ be given. Then there exists a closed nowhere dense set $A$ of irrational numbers in $[0,1[$ such that $\mu(A) \geqq 1-\delta$ and such that the sequences $(n a)_{n=1}^{\infty}(a \in A)$ are equi-uniformly distributed mod 1 .

Proof. For every integer $k \geqq 1$ we choose a real number $c_{k}>0$ in such a way that

$$
\mu\left\{x \in \left[0,1\left[:\left|e^{2 \pi i k x}-1\right|<c_{k}\right\} \leqq \frac{\delta}{2^{k}} .\right.\right.
$$

Let $A_{k}=\left\{x \in\left[0,1\left[:\left|e^{2 \pi i k x}-1\right| \geqq c_{k}\right\}\right.\right.$ and $\operatorname{let} A=\bigcap_{k=1}^{\infty} A_{k}$. Then $A$ is closed, consists of irrational numbers only, and we have $\mu(A) \geq 1-\sum_{k=1}^{\infty} \frac{\delta}{2^{k}}=1-\delta$. Furthermore, for any $k \geqq 1$ and for any $a \in A$ we have

$$
\left|\frac{1}{N} \sum_{n=1}^{N} e^{2 \pi i n k a}\right|=\frac{1}{N}\left|\frac{e^{2 \pi i N k a}-1}{e^{2 \pi i k a}-1}\right| \leqq \frac{2}{N c_{k}} .
$$

Thus, by Weyl's criterion, the sequences $(n a)_{n=1}^{\infty}(a \in A)$ are equi-uniformly distributed mod 1 and $A$ is nowhere dense by theorem 1 .

\section{Uniform distribution in eompact monothetic groups}

Let $X$ be a compact group which, as a topological space, is Hausdorff, and let $\mu$ be normed Haar measure on $X$. The group $X$ is called monothetic if there is at least one element $a \in X$ (called "generator") such that the sequence $\left(a^{n}\right)_{n=1}^{\infty}$ is everywhere dense in $X$ (VAN DaNTzIa [2]; cf. [4], [9] II $\S 9$ ). As a consequence, a compact monothetic group is abelian. If $a$ is a generator 
(and only in this case), the sequence $\left(a^{n}\right)_{n=1}^{\infty}$ is $\mu$-uniformly distributed [3]. If $\left(x_{n}\right)_{n=1}^{\infty}$ is any $\mu$-uniformly distributed sequence in $X$, then the sequences $\left(x_{n} y\right)(y \in X)$ are equi- $\mu$-uniformly distributed [10]. We denote by $\bar{A}$ the closure of a subset $A$ of $X$. In the rest of this section, we shall omit explicit reference to Haar measure $\mu$ in statements concerning uniform distribution.

Theorem 3. Let $A$ be $a$ set of generators of $X$ such that the sequences $\left(a^{n}\right)_{n=1}^{\infty}$ $(a \in A)$ are equi-uniformly distributed. Then also the sequences $\left(a^{n}\right)_{n=1}^{\infty}(a \in \bar{A})$ are equi-uniformly distributed.

Proof. Let $f \in C(X)$ and $\varepsilon>0$ be given. Suppose we have

$$
\left|\frac{1}{N} \sum_{n=1}^{N} f\left(a^{n}\right)-\mu(f)\right| \leqq \varepsilon \text { for all } N \geqq N(f, \varepsilon)
$$

and for all $a \in A$. Then, since $f\left(x^{n}\right)$ is a continuous function of $x$ for every $n$, (4) also holds for all $a \in \bar{A}$.

We note that theorem 3 yields another proof of theorem 1 which may be regarded as a corollary of theorem 3 . Indeed, if $A$ satisfies the hypotheses of theorem 1 and if $\bar{A}(\bmod 1)$ contained an open interval, then $\bar{A}$ would have to contain rational numbers which certainly are no generators. We may generalize the statement of theorem 1 in the following form:

Theorem 4. Let $X$ be not totally disconnected and let $A$ be a set of generators of $X$ such that the sequences $\left(a^{n}\right)_{n=1}^{\infty}(a \in A)$ are equi-uniformly distributed. Then $A$ is nowhere dense.

Proof. By Pontrjagin's duality theorem ([18] theorem 46, [9] theorem 24.26) there exists a continuous character $d$ of $X$ such that $d(X)=\{d(x): x \in X\}$ is the entire circle group $T$. By theorem 3, without loss of generality we may assume $A$ to be elosed. If $A$ contained an open subset of $X$ then $d(A)$ would have to contain an open interval in $T$ since $d$ is an open mapping of $X$ onto $T$. On the other hand, since $d$ is a continuous homomorphism, $d(A)$ would have to consist of generators of $T$ only, a contradiction.

Corollary 4.1. Suppose that $X$ is connected and $a$ is a generator of $X$. Then the sequences $\left(\left(a^{m}\right)^{n}\right)_{n=1}^{\infty}(m=1,2, \ldots)$ are uniformly distributed but not equiuniformly distributed.

Proof. Since $X$ is connected iff its dual group is torsion-free ([18] theorem 46, [9] theorem 24.25), we have $d\left(a^{m}\right)=d^{m}(a) \neq 1$ for every non-trivial character $d$ of $X$ and for every integer $m \geqq 1$. Thus, $a^{m}$ is a generator of $X$ ([9] theorem 25.11). The second assertion follows from theorem 4 since the sequence $\left(a^{m}\right)_{m=1}^{\infty}$ is everywhere dense in $X$.

We note that, by theorem 4, the second assertion of Corollary 4.1 even holds if $X$ is not totally disconnected, but in this case $a^{m}$ need not any more be a generator for every $m \geqq 2$. For convenience of notation we shall denote the set of all generators of $X$ by $G$.

Corollary 4.2. Suppose that $X$ is connected and satisfies the second axiom of countability. Then the family of all sequences $\left(a^{n}\right)_{n=1}^{\infty}(a \in G)$ is not the union of countably many families of equi-uniformly distributed sequences. 
Proof. Under the stated hypotheses, $G$ is a set of second category in $X$ ([4], ef. [9] 25.27).

Corollary 4.3. Let $X$ and $A$ be as in theorem 4. Then $\mu(\bar{A})<1$.

Proof. If $\mu(\bar{A})=1$, then $\bar{A}$ would have to be everywhere dense in $X$ since every open set has positive Haar measure.

If $X$ is totally disconnected, the assertion of theorem 4 may fail to hold. In fact, the sequences $\left(a^{n}\right)_{n=1}^{\infty}(a \in G)$ will be equi-uniformly distributed (see theorem 5 below) and $G$ may be open (see example following corollary 5.1 below). In order to show this we first establish an auxiliary result. We note that, in the absence of the second axiom of countability, Zorn's lemma is used in the proof.

Lemma 1. Let $d$ be a continuous character of $X$ having the property that $d(X)=T$. Then, for every irrational number $\alpha$ there exists a generator $a \in G$ such that $d(a)=e^{2 \pi i \alpha}$.

Proof. Let $D=\left\{d_{\lambda}: \lambda \in \Lambda\right\}$ be a complete system of different continuous characters of $X$, let $d_{0}$ be the trivial character $\left(d_{0} \equiv 1\right)$, and let $d_{1}=i$. Since $X$ is monothetic there is at least one generator $b \in G$. Suppose $d_{\lambda}(b)$ $=e^{2 \pi i \beta_{\lambda}}\left(\beta_{0}=1\right)$. By assumption $\left(d_{1}(X)=T\right), \beta_{1}$ is irrational. Let $T_{A}$ be the topological direct product $T_{A}=\prod_{\lambda \in \Lambda} T_{\lambda}\left(T_{\lambda}=T\right.$ for all $\left.\lambda \in \Lambda\right)$. It is well known that the mapping $\varphi: x \rightarrow \hat{x}=\left(d_{\lambda}(x)\right)_{\lambda \in A} \in T_{A}$ is a topological isomorphism of $X$ onto a closed subgroup $\hat{X}=\varphi(X)$ of $T_{A}$. In fact, $\hat{X}$ is the closure (with respect to the product topology in $T_{A}$ ) of the sequence $\left(\hat{b}^{n}\right)_{n=1}^{\infty} \subset T_{A}$. (The notation is chosen for technical reasons and should not be confused with the notation for residues mod 1 in section 2).

We now choose a maximal system of exponents $\beta_{\lambda}$, linearly independent over the field of rational numbers and including $\beta_{0}=1$ and $\beta_{1}$. We denote by $D^{\prime}=\left\{d_{\lambda^{\prime}}: \lambda^{\prime} \in \Lambda^{\prime}\right\}$ the corresponding subsystem of $D$. We now define an element $a$ of $T$ in the following way: first we choose arbitrarily a set of real numbers $\alpha_{\lambda^{\prime}}\left(\lambda^{\prime} \in \Lambda^{\prime}\right)$, linearly independent over the field of rational numbers and including $\alpha_{0}=1$ and $\alpha_{1}=\alpha$ (this may even be done by changing at most two of the numbers $\beta_{\lambda^{\prime}}$ ). Then we define $\alpha_{\lambda}$ for $\lambda \in \Lambda \backslash \Lambda^{\prime}$ by writing $\beta_{\lambda}$ as a finite linear combination of $\beta_{\lambda^{\prime}}$ 's with rational coefficients and replacing every $\beta_{\lambda^{\prime}}$ by $\alpha_{\lambda^{\prime}}$. Now we put $\hat{a}=\left(e^{2 \pi i \alpha_{\lambda}}\right)_{\lambda \in A} \in T_{A^{\prime}}$. If

$U\left(\hat{a} ; \lambda_{1}, \ldots, \lambda_{m} ; \varepsilon\right)=\left\{x=\left(e^{2 \pi i \xi_{\lambda}}\right)_{\lambda \in A} \in T_{\Lambda}:\left|e^{2 \pi i \xi_{\lambda_{k}}}-e^{2 \pi i \alpha_{\lambda_{k}}}\right|<\varepsilon, k=1, \ldots, m\right\}$

is an arbitrary neighbourhood of $\hat{a}$ in $T_{A}$, then we can find an integer $n$ (not necessarily positive) such that $\hat{b}^{n} \in U\left(\hat{a} ; \lambda_{1}, \ldots, \lambda_{m} ; \varepsilon\right)$. Indeed, because of our construction of $\alpha_{\lambda}$, for any lattice point $\left(h_{\lambda_{k}}\right)_{k=1}^{m}$, the congruence $\sum_{k=1}^{m} h_{\lambda_{k}} \beta_{\lambda_{k}} \equiv$ $0(\bmod 1)$ implies $\sum_{k=1}^{m} h_{\lambda_{k}} \alpha_{\lambda_{k}} \equiv 0(\bmod 1)$. Thus, by Kronecker's theorem ([14] VII $\$ 2$ ) the simultaneous inequalities

$$
\left|n \beta_{\lambda_{k}}-\alpha_{\lambda_{k}}\right|<\eta(\bmod 1) \quad(k=1, \ldots, m)
$$


have a common integral solution $n$ for any choice of $\eta=\eta(\varepsilon)$. Thus $a$ belongs to the group $\hat{X}$ and therefore there exists an element $a \in X$ such that $\hat{A}=\varphi(a)$.

By our construction $\alpha_{\lambda}$ will be an integer if and only if $\beta_{\lambda}$ is an integer. Since $b$ was a generator of $X$, this is the case if and only if $\lambda=0$. Thus, $d_{\lambda}(a) \neq 1$ for all $\lambda \neq 0$ and $a$ is a generator of $X$ ([9] theorem 25.11).

Theorem 5. The sequences $\left(a^{n}\right)_{n=1}^{\infty}(a \in G)$ are equi-uniformly distributed if and only if $X$ is totally disconnected.

Proof. We apply Weyl's criterion, taking in place of $F$ a complete system $D$ of different continuous characters of $X$. If $X$ is totally disconnected, then $d(X)$ is finite for every $d \in D$. For every $a \in G$ and for every non-trivial character $d \in D$, we therefore have $|d(a)-1| \geqq c_{d}>0$, where $c_{d}$ only depends on $d$. Thus, for every non-trivial character $d$, we obtain

$$
\left|\frac{1}{N} \sum_{n=1}^{N} d\left(a^{n}\right)\right|=\frac{1}{N}\left|\frac{d\left(a^{N+1}\right)-d(a)}{d(a)-1}\right| \leqq \frac{2}{N c_{d}} \leqq \varepsilon \text { for all } N \geqq \frac{2}{c_{d} \varepsilon}
$$

and for all $a \in G$.

If $X$ is not totally disconnected, then there is a character $d \in D$ such that $d(X)=T$. If the sequences $\left(a^{n}\right)_{n=1}^{\infty}(a \in G)$ were equi-uniformly distributed, then, by theorem 3 , we would have $G=\bar{G}$. In consequence, by lemma 1 , we would have $d(G)=T$. This, however, is impossible since $e^{2 \pi i \alpha}$ is not a generator of $T$ for rational $\alpha$.

Corollary 5.1. $G$ is closed if and only if $X$ is totally disconnected.

Proof. If $X$ is totally disconnected, the assertion follows from theorem 5 and theorem 3. If $X$ is not totally disconnected, the same reasoning applies as in the second part of the proof of theorem 5 .

Corollary 5.1 may of course also be proved without any reference to equiuniformly distributed sequences. As a consequence of lemma $1, G$ can certainly not be open unless $X$ is totally disconnected. If, however, this is the case, then $G$ may well be open as already demonstrated by any finite group with the discrete topology. Below we shall exhibit a compact totally disconnected monothetic group $X$ containing infinitely many elements and having an open set of generators. This, in connection with theorem 5 , shows that we cannot omit the hypothesis of $X$ not being totally disconnected in theorem 4 .

Let $T_{\infty}$ be the topological direct product of countably many copies of $T$. Let $a \in T_{\infty}$ be defined by $a=\left(e^{2 \pi i .2^{-k}}\right)_{k=1}^{\infty}$ and define $X$ to be the closure of the sequence $\left(a^{n}\right)_{n=1}^{\infty}$ in $T_{\infty}$. Then $X$ is a compact monothetic group ([9] theorem 9.16). Consider an element $b=\left(e^{2 \pi i b_{k} \cdot 2^{-k}}\right)_{k=1}^{\infty} \in X$ (b $b_{k}$ integers, $1 \leqq b_{k} \leqq 2^{k}$ ) with the property that $b_{1}=1$. Since $b$ may be approximated arbitrarily close by elements of the form $a^{n}(n \geqq 1)$, for every given integer $m \geqq 1$ the system of simultaneous congruences

$$
n \equiv b_{k}\left(\bmod 2^{k}\right) \quad(k=1, \ldots, m)
$$

has an integral solution $n>0$ which has to be an odd integer. Therefore, there exists an integer $n^{\prime}>0$ such that

$$
1 \equiv n^{\prime} b_{k}\left(\bmod 2^{k}\right) \quad(k=1, \ldots, m)
$$

and $a$ may be approximated arbitrarily close by elements of the form $b^{n}$. 
Thus, $b$ is a generator and we have $G=\left\{b=\left(e^{2 \pi i b_{k} \cdot 2^{-k}}\right)_{k=1}^{\infty}: b_{1}=1\right\}$. This set $G$, however, is obviously an open subset of $X$ of Haar measure $\frac{1}{2}$.

The following theorem establishes as generalization of theorem 2 which we, however, shall use in the proof. We note that, if $X$ satisfies the second axiom of countability, $G$ is a $G_{8}$-set and thus a Borel set ([9] 25.27).

Theorem 6. Suppose that $X$ satisfies the second axiom of countability and let $\varepsilon(0<\varepsilon<1)$ be given. Then there is a closed nowhere dense subset $Q$ of $G$ such that $\mu(Q)>\mu(G)-\varepsilon$ and such that the sequences $\left(a^{n}\right)_{n=1}^{\infty}(a \in Q)$ are equi. uniformly distributed.

Proof. Let $D=\left\{d_{k}: k=0,1,2, \ldots\right\}$ be a complete system of different continuous characters of $X$ and let $D^{\prime}$ be the subset of $D$ consisting of those characters $d$ for which $d(X)=T$ ( $D^{\prime}$ is empty iff $X$ is totally disconnected). We observe that, $\lambda$ denoting normed Haar measure on $T$, for every $d \in D^{\prime}$ and for every Borel set $B \subset T$, we have $\lambda(B)=\mu\left(d^{-1}(B)\right.$ ). (This may be seen e.g. by defining a Borel measure $\lambda$ on $T$ by this equation and checking that it has all the properties characterizing Haar measure on $T$ ). For every character $d_{k} \in D^{\prime}$ we may choose by theorem 2 a closed subset $A_{k}$ of $T$ having the property that the sequences $\left(d_{k}\left(a^{n}\right)\right)_{n=1}^{\infty}\left(d_{k}(a) \in A_{k}\right)$ are equi- $\lambda$-uniformly distributed in $T$ and such that $\lambda\left(A_{k}\right)>1-\frac{\varepsilon}{2^{k}}$. We put $Q_{1}=\bigcap_{d_{k} \in D^{\prime}} d_{k}^{-1}\left(A_{k}\right)$ if $D^{\prime}$ is not empty and $Q_{1}=X$ if $D^{\prime}$ is empty. Then $\mu\left(Q_{1}\right)>1-\varepsilon$ and, putting $Q=Q_{1} \cap G$, we have $\mu(Q)>\mu(G)-\varepsilon$.

Suppose now that $a \in Q$ is chosen arbitrarily and that $d_{k} \in D$ is any nontrivial character. If $d_{k}(X)=T$, then $d_{k}(a) \in A_{k}$ and

$$
\left|\frac{1}{N} \sum_{n=1}^{N} d_{k}\left(a^{n}\right)\right|=\left|\frac{1}{N} \sum_{n=1}^{N}\left(d_{k}(a)\right)^{n}\right| \leqq \varepsilon \text { for all } N \geqq N\left(d_{k}, \varepsilon\right)
$$

and for all $a \in Q$. If $d_{k}(X)$ is finite, then $d_{k}(a) \neq 1$ since $a$ is a generator and (6) holds by the reasoning used in the proof of (5). Thus, by Weyl's criterion, the sequences $\left(a^{n}\right)_{n=1}^{\infty}(a \in Q)$ are equi-uniformly distributed in $X$. By theorem 3 we may replace $Q$ by its closure which will then have the properties asserted in theorem 6.

We remark that, while no assumption of connectedness is necessary in the hypothesis, theorem 6 is interesting only in the non-totally disconnected case in view of theorem 5 .

Corollary 6.1. Suppose that $X$ is connected and satisfies the second axiom of countability. Then, for every given $\varepsilon(0<\varepsilon<1)$ there is a closed nowhere dense subset $A$ of $X$ such that $\mu(A)>1-\varepsilon$ and such that the sequences $\left(a^{n}\right)_{n=1}^{\infty}(a \in A)$ are equi-uniformly distributed.

Proof. Under the mentioned hypotheses we have $\mu(G)=1$ ([4], of. [9] 25.27). An application of theorem 6 completes the proof.

\section{Uniform distribution in compact Hausdorff spaces}

Let $X$ be any compact Hausdorff space and let $\mu$ be any regular normed Borel measure on $X$. Every sequence $\xi=\left(x_{n}\right)_{n=1}^{\infty} \subset X$ may be regarded as an 
element of the topological direct product space $X_{\infty}$ of countably many copies of $X$. We denote by $\overline{\mathfrak{A}}$ the closure of a subset $\mathfrak{U}$ of $X_{\infty}$ in the product topology. The theorems stated in the previous sections have rather close analoga also in the present context.

Theorem 7. Suppose that $\subseteq=\left\{\xi_{\sigma} \in X_{\infty}: \sigma \in S\right\}$ is a family of equi- $\mu$ uniformly distributed sequences. Then so is $\mathbb{E}$.

Proof. Let $f \in C(X)$ and $\varepsilon>0$ be given. By assumption, we have

$$
\left|\frac{1}{N} \sum_{n=1}^{N} f\left(x_{\sigma, n}\right)-\mu(f)\right| \leqq \varepsilon \text { for all } N \geqq N(f, \varepsilon)
$$

and for all $\sigma \in S$. Let $\xi=\left(x_{n}\right)_{n=1}^{\infty}$ be an arbitrary element of $X_{\infty}$ and define $F_{N}(\xi)=\frac{1}{N} \sum_{n=1}^{N} f\left(x_{n}\right)$. Then $F_{N}$ is a continuous function on $X_{\infty}$. Thus, for $\xi \in \overline{\mathcal{S}}$, we conclude from (7) that also

$$
\left|\frac{1}{N} \sum_{n=1}^{N} f\left(x_{n}\right)-\mu(f)\right| \leqq \varepsilon \text { for all } N \geqq N(f, \varepsilon) .
$$

This proves the assertion.

Theorem 8. Suppose that $X$ contains at least two points and that $\mathfrak{S}$ is a family of equi- $\mu$-uniformly distributed sequences. Then $\mathfrak{\Xi}$ is nowhere dense in $X$.

Proof. Assume the contrary. Because of theorem 7 we may assume that $\subseteq$ is closed and therefore contains an open subset $\mathcal{U}$ of $X_{\infty}$. If $X$ satisfies the second axiom of countability, then, via Baire's category theorem, this contradicts the well known fact that the set of all $\mu$-uniformly distributed sequences in $X$ is of first category in $X_{\infty}$ ([12] Satz 12).

If $X$ does not satisfy the second axiom of countability we may argue in the following way: Without loss of generality we may assume that $\mathfrak{U}$ is of the form $\mathfrak{U}=\prod_{n=1}^{\infty} U_{n}$, where $U_{n}$ is an open subset of $X$ for all $n \geqq 1$ and $U_{n}=X$ for all $n \geqq N_{1}$. Let $V$ and $W$ be open subsets of $X$ with disjoint closures. Let $f$ be a Urysohn function on $X$ (i.e. $f \in C(X)$ and $0 \leqq f(x) \leqq 1$ for all $x \in X$ ) such that $f(x)=1$ for all $x \in V$ and $f(x)=0$ for all $x \in W$. Let $N \geqq 3 N_{1}$ be given. Because of our assumption $\mathfrak{S}$ contains sequences $\eta=\left(y_{n}\right)_{n=1}^{\infty} \in \mathcal{U}$ and $\zeta=\left(z_{n}\right)_{n=1}^{\infty} \in \mathcal{U}$ with the following properties: $y_{n} \in V$ for $N_{1}<n \leqq N$ and $z_{n} \in W$ for $N_{1}<n \leqq N$. We conclude $\frac{1}{N} \sum_{n=1}^{N} f\left(y_{n}\right) \geqq \frac{2}{3}$ and $\frac{1}{N} \sum_{n=1}^{N} f\left(z_{n}\right) \leqq \frac{1}{3}$. Since $N$ was arbitrary, this contradicts our assumption that $\mathscr{E}$ is a family of equi- $\mu$-uniformly distributed sequences.

If $X$ satisfies the second axiom of countability, then, as mentioned above, the set of all $\mu$-uniformly distributed sequences in $X$ is of first category in $X_{\infty}$. This could suggest the idea that, in contrast to corollary 1.2 and 4.2, the set of all $\mu$-uniformly distributed sequences might well be the countable union of families of equi- $\mu$-uniformly distributed sequences. A closer inspection, however, shows that this still will not be the case in general. Indeed, if $X$ is a compact connected abelian group satisfying the second axiom of countabi- 
lity (and a fortiori monothetic [9] 25.14) and if $\mu$ is normed Haar measure on $X$, then the set of all $\mu$-uniformly distributed sequences in particular contains all sequences $\left(a^{n}\right)_{n=1}^{\infty}(a \in G)$. This set, however, cannot be split into the union of countably many families of equi- $\mu$-uniformly distributed sequences by corollary 4.2 .

In order to state an analogon of corollary 4.1 (cf. [8] theorem 2) we recall that the shift transformation $P$ in $X_{\infty}$ is defined by $P\left(x_{n}\right)_{n=1}^{\infty}=\left(y_{n}\right)_{n=1}^{\infty}$, where $y_{n}=x_{n+1}(1 \leqq n<\infty)$.

Corollary 8.1. Suppose that $X$ contains at least two points. Suppose furthermore that $\xi \in X_{\infty}$ is $\mu$-uniformly distributed in $X$ and that, for some subsequence $\left(m_{k}\right)_{k=1}^{\infty}$ of the positive integers, the sequence $\left(P^{m_{k}} \xi_{k=1}^{\infty}\right.$ is dense in some open subset of $X_{\infty}$. Then the sequences $P^{m_{k}} \xi(k=1,2, \ldots)$ are $\mu$-uniformly distributed but not equi- $\mu$-uniformly distributed.

Corollary 8.2. Let $X$ and $\subseteq$ be as in theorem 8 and suppose that $X$ is the support of $\mu$. Then $\mu_{\infty}$ (E) $<1$.

Proof. Under the mentioned hypothesis, the support of $\mu_{\infty}$ is $X_{\infty}$. If $\bar{E}$ had $\mu_{\infty}$-measure 1 , then $\bar{S}$ would coincide with $X_{\infty}$, in contradiction to theorem 8 .

The following theorem and its proof are essentially due to E. HrawkA ([10] $\S 6$ ) who has stated them for the case of a compact group.

Theorem 9. Suppose that $X$ satisfies the second axiom of countability and let $\delta(0<\delta<1)$ be given. Then there exists a closed family $\lesssim$ of equi- $\mu$-uniformly distributed sequences such that $\mu_{\infty}(\mathfrak{E})>1-\delta$.

Proof. In order to be able to apply Weyl's criterion we first note that because of the second axiom of countability there exists a countable set $F=\left\{f_{k}: k=1,2, \ldots\right\} \subset C(X)$ as described in section 1 . Given any $k \geqq 1$ we again define $F_{k, N} \in C\left(X_{\infty}\right)$ for $\xi=\left(x_{n}\right)_{n=1}^{\infty}$ by

$$
F_{k, N}(\xi)=\frac{1}{N} \sum_{n=1}^{\infty} f_{k}\left(x_{n}\right) \text {. }
$$

It is a well known consequence of the individual ergodic theorem ([6], [10] $\$ 6$, $[12] \S 3$ ) that

$$
\lim _{N \rightarrow \infty} F_{k, N}(\xi)=\mu\left(f_{k}\right)
$$

for $\mu_{\infty}$-almost all $\xi \in X_{\infty}$. Therefore, by Egoroff's theorem ([5] $\S 21 \mathrm{~A}$ ), there exists a measurable subset $\Xi_{k}$ of $X_{\infty}$ of measure $\mu_{\infty}\left(\digamma_{k}\right) \geqq 1-\frac{\delta}{2^{k}}$ such that the functions $F_{k, N}$ converge uniformly on $\varsigma_{k}$ for $N \rightarrow \infty$. Taking $\varsigma_{0}=\bigcap_{k=1}^{\infty} \varsigma_{k}$ we obtain $\mu_{\infty}\left(\mathcal{S}_{0}\right) \geqq 1-\delta$. Furthermore, for every $k \geqq 1$ and for every given $\varepsilon>0$, we have

$$
\left|\frac{1}{N} \sum_{n=1}^{N} f_{k}\left(x_{n}\right)-\mu\left(f_{k}\right)\right| \leqq \varepsilon \text { for all } N \geqq N\left(f_{k}, \varepsilon\right)
$$

and for all $\xi=\left(x_{n}\right)_{n=1}^{\infty} \in \mathcal{G}=\overline{\mathcal{E}}_{0}$. An application of Weyl's criterion leads to the assertion. 


\section{Almost well distributed sequences}

Let $X$ be a compact Hausdorff space and let $\mu$ be a regular normed Borel measure on $X$. As in the preceding section we denote by $P$ the shift transformation in infinite product space $X_{\infty}$. A sequence $\xi \in X_{\infty}$ is called $\mu$-well distributed if the sequences $\boldsymbol{P}^{m} \boldsymbol{\xi}(m=1,2, \ldots)$ are equi- $\mu$-uniformly distributed (Huawka [10], Petersen [17]). While under the assumption of the second axiom of countability $\mu_{\infty}$-almost every sequence is $\mu$-uniformly distributed ([12] Satz 11), $\mu_{\infty}$-almost no sequence is $\mu$-well distributed if $\mu$ is not a point-measure ([8] corollary 2.1). We shall now apply the results of the preceeding section to some questions connected with the concept of good distribution.

Theorem 10. Suppose that the sequence $\xi \in X_{\infty}$ is $\mu$-well distributed. Then so ist every sequence in the closure of the set $\mathfrak{S}=\left\{P^{m} \xi: m=1,2, \ldots\right\}$.

Proof. By theorem $7, \overrightarrow{\mathcal{S}}$ is a family of equi- $\mu$-uniformly distributed sequences. If $\eta$ belongs to $\overline{\mathcal{E}}$, then so does $P^{m} \eta$ for every $m \geqq 1$.

As an illustration in the case of uniform distribution $\bmod 1$ we note that, if $a$ is irrational and if $\xi$ is the sequence $(n a)_{n=1}^{\infty}(\bmod 1)$, then $\vec{E}$ is the family of all sequences $(n a+b)_{n=1}^{\infty}(\bmod 1)(b \in[0,1[)$.

We shall call a sequence $\xi \in X_{\infty}$ almost $\mu$-well distributed if there exists an infinite subsequence $\left(m_{k}\right)_{k=1}^{\infty}$ of the non-negative integers such that the sequences $P^{m_{k}} \xi(k=1,2, \ldots)$ are equi- $\mu$-uniformly distributed. If we want to refer to this particular subsequence we shall call $\xi$ almost $\mu$-well distributed $\left(m_{k}\right)_{k=1}^{\infty}$. Note that the very definition implies that an almost $\mu$-well distributed sequence is $\mu$-uniformly distributed.

Theorem 11. Suppose that $\xi \in X_{\infty}$ is almost $\mu$-well distributed $\left(m_{k}\right)_{k=1}^{\infty}$ and let $h$ be any positive integer. Then $\xi$ is also almost $\mu$-well distributed $\left(m_{k}+h\right)_{k=1}^{\infty}$.

Proof. For any given $f \in C(X)$ and for any $\varepsilon>0$ we have

$$
\left|\frac{1}{N} \sum_{n=m_{k}+1}^{m_{k}+N} f\left(x_{n}\right)-\mu(f)\right| \leqq \varepsilon \text { for all } N \geqq N(f, \varepsilon)
$$

and for all $k \geqq 1$. We conclude

$$
\begin{aligned}
\left|\frac{1}{N} \sum_{n=m_{k}+h+1}^{m_{k}+h+N} f\left(x_{n}\right)-\mu(f)\right| & \leqq\left|\frac{1}{N} \sum_{n=m_{k}+N+1}^{m_{k}+N+h} f\left(x_{n}\right)-\sum_{n=m_{k}+1}^{m_{k}+h} f\left(x_{n}\right)\right|+ \\
& +\left|\frac{1}{N} \sum_{n=m_{k}+1}^{m_{k}+N} f\left(x_{n}\right)-\mu(f)\right| \leqq \\
& \leqq \frac{2 h \| f f \mid}{N}+\varepsilon \leqq 2 \varepsilon \text { for all } N \geqq \max \left(N(f, \varepsilon), \frac{2 h\|f\|}{\varepsilon}\right)
\end{aligned}
$$

and for all $k \geqq 1$.

Corollary 11.1. Let $\xi$ and $h$ be as in theorem 11. Then the sequences $P^{m_{k}+m} \xi$ $(|m| \leqq h, k=1,2, \ldots)$ are equi- $\mu$-uniformly distributed.

Proof. By theorem 11, for every fixed $m$, the sequences $P^{m_{k}+m} \xi(k=1,2, \ldots)$ are equi- $\mu$-uniformly distributed. Taking the union of these finitely many families of sequences, we again obtain a family of equi- $\mu$-uniformly distributed sequences. 
Corollary 11.2. Suppose that $\xi \in X_{\infty}$ is almost $\mu$-vell distributed $\left(m_{k}\right)_{k=1}^{\infty}$ and that the differences $\left(m_{k+1}-m_{k}\right)$ are bounded. Then $\xi$ is $\mu$-well distributed.

Proof. Put $m_{0}=0$, let $h=\max _{k \geq 0}\left(m_{k+1}-m_{k}\right)$ and apply corollary 11.1 .

If we compare these statements with the results of the previous sections, we may regard corollary 11.1 as some sort of analogon to theorem 3 and theorem 7. In fact, it asserts that, restricting attention to the subsequences $P^{m} \xi$ of a given $\mu$-uniformly distributed sequence $\xi$, every family of equi- $\mu$-uniformly distributed subsequences $P^{m_{k}} \xi(k=1,2, \ldots)$ may be enlarged by an operation similar to taking the closure, referring, however, not to a topology but to the concept of relative density of a set of integers. In this sense, corollary 11.2 appears to be an analogon to theorem 4 and theorem 8 if we state it in the following form: Suppose that $\xi \in X_{\infty}$ is not $\mu$-well distributed and suppose that the sequences $P^{m_{k}} \xi(k=1,2, \ldots)$ are equi- $\mu$-uniformly distributed. Then the sequence of integers $\left(m_{k}\right)_{k=1}^{\infty}$ is not relatively dense (i.e. it is not possible to find a positive integer $h$ such that every interval of length $h$ contains at least one integer $m_{k}$ ).

The following theorem again stems from a remark of E. HLAwKA ([10] last paragraph; the statements "jede Folge" and "offene Menge" in the passage referred to are erroneous). In the context just mentioned, it establishes an analogon to theorem 6 and theorem 9.

Theorem 12. Suppose that $X$ satisfies the second axiom of countability. Then $\mu_{\infty}$-almost every sequence $\xi \in X_{\infty}$ is almost $\mu$-well distributed. In particular, $\mu_{\infty}$-almost every sequence $\xi \in X_{\infty}$ has the following property: given any $\delta(0<\delta<1)$ there exists a subsequence $\left(m_{k}\right)_{k=1}^{\infty}$ of the non-negative integers of asymptotic density greater than $1-\delta$ such that $\xi$ is almost $\mu$-well distributed $\left(m_{k}\right)_{k=1}^{\infty}$.

Proof. By theorem 9 we can find a sequence of families $\tilde{S}_{j}$ of equi- $\mu$-uniformly distributed sequences such that $\mu_{\infty}\left(\mathscr{\sigma}_{j}\right) \geqq 1-\frac{1}{j}(j=1,2, \ldots)$. We note that $\boldsymbol{P}$ is a $\mu_{\infty}$-measure preserving ergodic transformation in $X_{\infty}^{-}$[6]. By the individual ergodic theorem, there is a subset $\mathfrak{T}_{j}$ of $X_{\infty}$ such that $\mu_{\infty}\left(\mathfrak{I}_{j}\right)=1$ and such that for every $\xi \in \mathfrak{T}_{j}$ the sequence of exponents $m_{k}$ for which $P^{m_{k}} \xi \in \mathscr{S}_{j}$ has asymptotic density $\mu_{\infty}\left(\mathscr{E}_{j}\right)$. Taking $\mathfrak{T}=\prod_{j=1}^{\infty} \mathfrak{T}_{j}$ we obtain $\mu_{\infty}(\mathfrak{I})=1$ and every $\xi \in \mathfrak{T}$ has the property stated in the theorem.

6. Almost well, weakly well, and completely uniformly distributed sequences

Let, as in section $5, X$ be a compact Hausdorf space and let $\mu$ be a regular normed Borel measure on $X$. In view of theorem 12 we are led to consider the following classes of sequences in $X$ and to ask for the set-theoretic and measure-theoretic relations between them:

$\mathfrak{U}=\left\{\xi \in X_{\infty}^{-}: \xi\right.$ is $\mu$-uniformly distributed $\}$.

$\mathfrak{A W}=\left\{\xi \in X_{\infty}: \xi\right.$ is almost $\mu$-well distributed $\}$.

$\mathscr{U} \mathfrak{W}_{1}=\left\{\xi \in X_{\infty}^{\infty}:\right.$ for every $\delta(0<\delta<1)$ there exists a subsequence $\left(m_{k}\right)_{k=1}^{\infty}$ of the non-negative integers of asymptotic density greater than $1-\delta$ such that $\xi$ is almost $\mu$-well distributed $\left.\left(m_{k}\right)_{k=1}^{\infty}\right\}$. 
$\mathfrak{A W}_{2}=\left\{\xi \in X_{\infty}\right.$ : there exists a subsequence $\left(m_{k}\right)_{k=1}^{\infty}$ of the non-negative integers of asymptotic density 1 such that $\xi$ is almost $\mu$-well distributed $\left.\left(m_{k}\right)_{k=1}^{\infty}\right\}$.

$$
\mathfrak{B}=\left\{\xi \in X_{\infty}: \xi \text { is } \mu \text {-well distributed }\right\} \text {. }
$$

Obviously we have $\mathfrak{U} \supset \mathfrak{A W}>\mathfrak{A} \mathfrak{W}_{1}>\mathfrak{A} \mathfrak{W}_{2} \supset \mathfrak{W}$. Furthermore, if the second axiom of countability is satisfied, we have $\mu_{\infty}\left(\mathfrak{A} \mathfrak{W}_{1}\right)=1$ by theorem 12 and $\mu_{\infty}(\mathfrak{B})=0$ if $\mu$ is not a point measure by [8] corollary 2.1. In what follows we shall show that, under these hypotheses and in the presence of $\mu$-well distributed sequences, each of the above inclusions is strict and $\mu_{\infty}\left(\mathfrak{A} \mathfrak{W}_{2}\right)=0$.

In this context it seems appropriate also to clarify the relations between the properties of a sequence $\xi \in X_{\infty}$ to be almost $\mu$-well distributed, weakly $\mu$-well distributed in the sense of HLAwKa [11] and completely $\mu$-uniformly distributed in the sense of Кововоv [15] (cf. [13] § 4). We shall therefore also consider the following classes of sequences in $X$ :

$\mathfrak{W} \mathfrak{W}=\left\{\xi \in X_{\infty}: \xi\right.$ is weakly $\mu$-well distributed, i.e. for every $f \in C(X)$ we have

$$
\left.\lim _{N \rightarrow \infty} \limsup _{R \rightarrow \infty} \frac{1}{R} \sum_{r=0}^{R-1}\left|\frac{1}{N} \sum_{n=r N+1}^{(r+1) N} f\left(x_{n}\right)-\mu(f)\right|=0\right\} .
$$

$\mathfrak{C} \mathcal{U}=\left\{\xi \in X_{\infty}: \xi\right.$ is completely $\mu$-uniformly distributed, i.e. the sequence $\left(P^{m} \xi\right)_{m=1}^{\infty}$ is $\mu_{\infty}$-uniformly distributed in $\left.X_{\infty}\right\}$.

Again, it is well known and easy to see that we have $\mathfrak{U} \supset \mathfrak{s} \mathfrak{U}$ and $\mathfrak{U} \supset \mathfrak{W} \mathfrak{W} \supset \mathfrak{W}$. Furthermore, if the second axiom of countability is satisfied, we have $\mu_{\infty}(\mathfrak{E} \mathfrak{U})=1$ (replacing in the corresponding statement about uniformly distributed sequences $X$ by $X_{\infty}$ and $\mu$ by $\left.\mu_{\infty}\right), \mu_{\infty}(\mathfrak{D B D})=1$ by [11] Satz 2 (cf. theorem 16), and $(\mathcal{U} \cap \mathfrak{W}=\emptyset$ by [8] theorem 2 if $\mu$ is not a point measure. Thus, $\mu_{\infty}$-almost every sequence $\xi \in X_{\infty}$ is at the same time almost $\mu$-well distributed (even in the sense of $\mathfrak{\mathscr { W }} \mathfrak{W}_{1}$ ), weakly $\mu$-well distributed, and completely $\mu$-uniformly distributed. We shall show that, in fact, $\mathfrak{C} \mathfrak{U} \cap \mathfrak{A W}_{2}=\emptyset$ if $\mu$ is not a point measure, thus establishing $\mu_{\infty}\left(\mathfrak{A F}_{2}\right)=0$, and that, in the presence of $\mu$-well distributed sequences, all inclusions in the chain $\mathfrak{U} \supset \mathfrak{W} \mathfrak{B} \supset \mathfrak{W}$ are strict. In particular, we have $\mathfrak{W} \mathfrak{W} \supset \mathfrak{A F}_{2}$ but $\mathfrak{W} \mathfrak{W} D \mathfrak{A W}$. Part of the proofs will be carried out by explicitly constructing sequences that are of one type but not of the other.

Let us note again that, in the definition of weakly $\mu$-well distributed sequences, we can replace $C(X)$ by the larger class of functions described at the end of section 1. In fact, if $\xi \in X_{\infty}$ is weakly $\mu$-well distributed and if $f, f_{1}, f_{2}$ are real-valued Borel measurable functions $\left(f_{j} \in C(X), j=1,2\right)$ satisfying $f_{1} \leqq f \leqq f_{2}$ and $\mu\left(f_{2}-f_{1}\right) \leqq \frac{\varepsilon}{2}$, then we can choose $N(f, \varepsilon)$ so large that for all $N \geqq N(f, \varepsilon)$ we have

$$
\limsup _{R \rightarrow \infty} \frac{1}{R} \sum_{r=0}^{R-1}\left|\frac{1}{N} \sum_{n=r+N+1}^{(r+1) N} f_{j}\left(x_{n}\right)-\mu\left(f_{j}\right)\right| \leqq \frac{\varepsilon}{4} \quad(j=1,2) .
$$


By (2) we conclude

$$
\begin{gathered}
\limsup _{R \rightarrow \infty} \frac{1}{R} \sum_{r=0}^{R-1}\left|\frac{1}{N} \sum_{n=r+N+1}^{(r+1) N} f\left(x_{n}\right)-\mu(f)\right| \leqq \\
\leqq \sum_{j=1}^{2} \limsup _{R \rightarrow \infty} \frac{1}{R} \sum_{r=0}^{R-1}\left|\frac{1}{N} \sum_{n=r N+1}^{(r+1) N} f_{j}\left(x_{n}\right)-\mu\left(f_{j}\right)\right|+\frac{\varepsilon}{2} \leqq \varepsilon
\end{gathered}
$$

This proves our assertion.

We now proceed with the detailed exposition of the statements outlined above. The proof of the following theorem is, with some slight modifications, the same as the proof of theorem 2 in [8]. We shall, however, reproduce it here for the convenience of the reader.

Theorem 13. Suppose that $\mu$ is not a point measure and suppose that $\xi=\left(x_{n}\right)_{n=1}^{\infty} \in X_{\infty}$ is completely $\mu$-uniformly distributed. Then $\xi$ is not almost $\mu$-well distributed with respect to any subsequence $\left(m_{k}\right)_{i=1}^{\infty}$ of the non-negative integers of asymptotic density 1 .

Proof. Assume the contrary and let $\mathfrak{N}=\left(m_{k}\right)_{k=1}^{\infty}$ be a subsequence of the non-negative integers of asymptotic density 1 such that $\xi$ is almost $\mu$-well distributed $\left(m_{k}\right)_{k=1}^{\infty}$. Since $\mu$ is not concentrated in one point we can find an open set $A \subset X$ such that $0<\mu(A)<1$. Without loss of generality we may assume that the boundary of $A$ has $\mu$-measure zero. Let $N \geqq 1$ be given and let $A_{\infty}=\prod_{n=1}^{\infty} A_{n}$ where $A_{n}=A$ for $1 \leqq n \leqq N$ and $A_{n}=X$ for $n>N$. Then $A_{\infty}$ is open in $X$ and its boundary has $\mu_{\infty}$-measure zero. Furthermore, we have $0<\mu_{\infty}\left(A_{\infty}\right)<1$. Since the sequence $\left(P^{m} \xi\right)_{m=1}^{\infty}$ is by assumption $\mu_{\infty}$-uniformly distributed in $X_{\infty}$, and since $\mathfrak{M}$ has asymptotic density 1 , so is the sequence $\left(P^{m_{k}} \xi\right)_{k=1}^{\infty}$. Therefore, there exists a positive integer $k(N)$ such that $P^{m_{k}(N)} \xi \in A_{\infty}$. Hence, for every choice of $N \geqq 1$, we have

$$
\frac{1}{N} \sum_{n=m_{k(N)}+1}^{m_{k(N)}+N} \chi_{A}\left(x_{n}\right)-\mu(A)=1-\mu(A)>0 .
$$

This, however, contradicts our assumption that $\xi$ is almost $\mu$-well distributed $\left(m_{k}\right)_{k=1}^{\infty}$. (If the support of $\mu$ is $X$ the theorem can also be derived from corollary 8.1).

Corollary 13.1. Suppose that $X$ satisfies the second axiom of countability and that $\mu$ is not a point measure. Then $\mu_{\infty}\left(\mathfrak{A P}_{2}\right)=0$.

Proof. Under the mentioned hypothesis, we have $\mu_{\infty}(\mathfrak{E} \mathfrak{U})=1$. The statement then follows from theorem 13.

Corollary 13.2. Suppose that $X$ satisfies the second axiom of countability and that $\mu$ is not a point measure. Then $\mathfrak{A W}_{1} \supsetneqq \mathfrak{A W}_{2}$.

Proof. The statement follows from theorem 12 and corollary 13.1.

Theorem 14. Suppose that $X$ contains at least two points and that there exists $a \mu$-well distributed sequence. Then there also exists a sequence $\xi \in X_{\infty}$, constructed explicitely below, which is not $\mu$-well distributed but almost $\mu$-well distributed $\left(m_{k}\right)_{k=1}^{\infty}$ where $\left(m_{k}\right)_{k=1}^{\infty}$ has asymptotic density 1 . Consequently we have $\mathfrak{A W} \mathfrak{2} \nsucceq \mathfrak{W}$. 
Proof. Suppose that the sequence $\eta=\left(y_{k}\right)_{k=1}^{\infty} \in X_{\infty}$ is $\mu$-well distributed and let $a \in X$ belong to the support of $\mu$. We then choose a point $b \neq a$ in $X$ and construct a sequence $\xi=\left(x_{n}\right)_{n=1}^{\infty} \in X_{\infty}$ by induction as follows: Let $x_{1}=b$. If $x_{1}, \ldots, x_{h^{4}}(h \geqq 1$ an integer) have already been defined such as to comprise all elements $y_{k}$ with $k \leqq h^{4}-h(2 h-1)$, then let $x_{n}\left(h^{4}<n \leqq h^{4}+4 h^{3}+6 h^{2}\right)$ be the next $4 h^{3}+6 h^{2}$ consecutive $y_{k}{ }^{3}$ s and let $x_{n}=b$ for $h^{4}+4 h^{3}+6 h^{2}<$ $<n \leqq(h+1)^{4}$.

Obviously, due to the increasing stretches of $b$ 's which certainly do not cover the support of $\mu$, the sequence $\xi$ is not $\mu$-well distributed. If we allow $m$ to run through all integers satisfying $h^{4} \leqq m<h^{4}+4 h^{3}(h=1,2, \ldots)$, then it is easy to see that this subsequence $\mathfrak{R}$ of the positive integers has asymptotic density 1 . We shall now show that the corresponding sequences $P^{m} \xi(m \in \mathfrak{M})$ are equi- $\mu$-uniformly distributed.

To this purpose, let $f \in C(X)(f \neq 0)$ and $\varepsilon>0$ be given and suppose that

$$
\left|\frac{1}{N} \sum_{n=m+1}^{m+N} f\left(y_{n}\right)-\mu(f)\right| \leqq \frac{\varepsilon}{2} \text { for all } N \geqq N(f, \varepsilon)
$$

and for all $m=1,2, \ldots$ It will suffice to show that

$$
\left|\frac{1}{N} \sum_{n=m+1}^{m+N} f\left(x_{n}\right)-\mu(f)\right| \leqq \varepsilon \text { for all } N \geqq N(f, \varepsilon)
$$

and for all $m \in \mathfrak{R}$ which are greater than the fourth power of some fixed integer $H=H(f, \varepsilon)$. In fact, we choose this integer $H$ in such a way that $\frac{4 H+1}{6 H^{2}+4 H+1}<$ $<\frac{\varepsilon}{4\|f\|}$ and $6 H^{2} \geqq N(f, \varepsilon)$. For a fixed $m \in \mathfrak{R}, m \geqq H^{4}$, let us denote by $N^{\prime}$ the number of elements $x_{m+n}(1 \leqq n \leqq N)$ chosen from the sequence $\eta$ and by $\Sigma^{\prime} f\left(x_{n}\right)$ the sum over these elements. For all $N \geqq N(f, \varepsilon)$ we have $N^{\prime} \geqq N(f, \varepsilon)$ because of $h^{4} \leqq m<h^{4}+4 h^{3}$ and $6 h^{2} \geqq 6 H^{2} \geqq N(f, \varepsilon)$. Thus we obtain

$$
\begin{aligned}
\frac{1}{N} \sum_{n=m+1}^{m+N} f\left(x_{n}\right) & =\frac{N^{\prime}}{N} \cdot \frac{1}{N^{\prime}} \Sigma^{\prime} f\left(x_{n}\right)+\frac{N-N^{\prime}}{N} f(b) \\
& =\frac{1}{N^{\prime}} \Sigma^{\prime} f\left(x_{n}\right)-\frac{N-N^{\prime}}{N} \cdot \frac{1}{N^{\prime}} \Sigma^{\prime} f\left(x_{n}\right)+\frac{N-N^{\prime}}{N} f(b)
\end{aligned}
$$

and

$$
\begin{gathered}
\left|\frac{1}{N} \sum_{n=m+1}^{m+N} f\left(x_{n}\right)-\mu(f)\right| \leqq\left|\frac{1}{N^{\prime}} \Sigma^{\prime} f\left(x_{n}\right)-\mu(f)\right|+2 \frac{N-N^{\prime}}{N}\|f\| \leqq \\
\leqq \frac{\varepsilon}{2}+2 \frac{N-N^{\prime}}{N}\|f\| .
\end{gathered}
$$

Because of our construction of $\xi$ and of the restriction imposed on $m$ we have

$$
\frac{N-N^{\prime}}{N} \leqq \frac{4 h+1}{6 h^{2}+4 h+1} \leqq \frac{4 H+1}{6 H^{2}+4 H+1} \leqq \frac{\varepsilon}{4\|f\|} .
$$

This, in connection with (9), proves (8).

In order to establish the inclusion $\mathfrak{W} \mathfrak{W}>\mathfrak{A P}_{2}$ we again need a lemma. 
Lemma 2. Let $\mathfrak{M}$ be a subsequence of the non-negative integers of asymptotic density 1 and let $N$ be given. Then the subsequence $\mathfrak{R}=\{r: r N \in \mathfrak{R}\}$ of the non-negative integers also has asymptotic density 1.

Proof. For any integer $M \geqq 1$ we define

$$
v(\mathfrak{R}, M)=\sum_{\substack{m \in \mathfrak{R} \\ m<M}} 1
$$

(for other sequences, the counting function $\nu$ is defined analogously). Suppose $\liminf _{R \rightarrow \infty} \frac{v(R, R)}{R}=1-2 \delta(\delta>0)$, contrary to the assertion. We then have

$$
\nu(\mathfrak{R}, R N) \leqq v(R, R)+(N-1) R \quad \text { for every } R \geqq 1
$$

and therefore

$$
\frac{\nu(\mathfrak{M}, R N)}{R N} \leqq \frac{1}{N} \cdot \frac{\nu(\Re, R)}{R}+\frac{N-1}{N}<\frac{1}{N}(1-\delta)+\frac{N-1}{N}=1-\frac{\delta}{N}
$$

for infinitely many $R$, in contradiction to our assumption.

Theorem 15. Suppose that the sequence $\xi=\left(x_{n}\right)_{n=1}^{\infty} \in X_{\infty}$ is almost $\mu$-well distributed $\left(m_{k}\right)_{k=1}^{\infty}$ and $\mathfrak{M}=\left(m_{k}\right)_{k=1}^{\infty}$ has asymptotic density 1 . Then $\xi$ is weakly $\mu$-well distributed. Consequently, we have $\mathfrak{W} \mathfrak{B} \supset \mathfrak{A W}_{2}$.

Proof. Let $f \in C(X)$ and $\varepsilon>0$ be given and let

$$
\left|\frac{1}{N} \sum_{n=m+1}^{m+N} f\left(x_{n}\right)-\mu(f)\right| \leqq \varepsilon \text { for all } N \geqq N(f, \varepsilon)
$$

and for all $m \in \mathfrak{R}$. Fix any $N \geqq N(f, \varepsilon)$ and let $R=\{r \geqq 0: r N \in \mathfrak{M}\}$. Furthermore, let $\mathfrak{R}^{\prime}$ be the subsequence of the non-negative integers, complementary to $\Re$. Then we have

$$
a_{N, r}=\left|\frac{1}{N} \sum_{n=r N+1}^{(r+1) N} f\left(x_{n}\right)-\mu(f)\right| \leqq \varepsilon \text { for all } r \in \mathfrak{R}
$$

and $a_{N, r} \leqq 2\|f\|$ for all $r \geqq 0$. Since $\Re$ has asymptotic density 1 by lemma 2 , $\mathfrak{R}^{\prime}$ has asymptotic density 0 . We conclude

$$
\begin{aligned}
\frac{1}{R} \sum_{r=0}^{R-1} a_{N, r} & =\frac{1}{R}\left(\sum_{\substack{r \in \mathbb{R}^{\prime} \\
r<R}} a_{N, r}+\sum_{\substack{r \in \mathscr{R}^{\prime} \\
r<R}} a_{N, r}\right) \leqq \\
& \leqq \frac{R \varepsilon}{R}+\frac{\nu\left(R^{\prime}, R\right)}{R} \cdot 2\|f\| \leqq 2 \varepsilon \text { for all } R \geqq R_{0} .
\end{aligned}
$$

As a consequence, we have

$$
\limsup _{R \rightarrow \infty} \frac{1}{R} \sum_{r=0}^{R-1} a_{N, r} \leqq 2 \varepsilon \text { for all } N \geqq N(f, \varepsilon)
$$

and, since $\varepsilon$ was arbitrary,

$$
\lim _{N \rightarrow \infty} \limsup _{R \rightarrow \infty} \frac{1}{R} \sum_{r=0}^{R-1}\left|\frac{1}{N} \sum_{n=r N+1}^{(r+1) N} f\left(x_{n}\right)-\mu(f)\right|=0 .
$$

For the case of a compact group $X$ with normed Haar measure $\mu$, the following theorem has been proved by HLAwKA ([11] Satz 2). In order to obtain an even sharper statement, the proof given there actually makes use of group 
theoretic concepts. We shall therefore give another proof here which makes use of ergodic theory and is valid also in the more general situation considered in this section (the possibility of this approach has also already been mentioned by HLAwkA).

Theorem 16. Suppose that $X$ satisfies the second axiom of countability. Then $\mu_{\infty}$-almost all sequences $\xi \in X_{\infty}$ are weakly $\mu$-well distributed.

Proof. We note that Weyl's criterion holds for weakly $\mu$-well distributed sequences just as well. We therefore choose a countable subset $F=\left\{f_{k}: k\right.$ $=1,2, \ldots\} \subset C(X)$ as described in section 1 and fix a function $f=f_{k} \in F^{\prime}(f \neq 0)$. We then define the function $F_{N} \in C\left(X_{\infty}\right)$ for $\xi=\left(x_{n}\right)_{n=1}^{\infty}$ by

$$
F_{N}(\xi)=\left|\frac{1}{N} \sum_{n=1}^{N} f\left(x_{n}\right)-\mu(f)\right| .
$$

Since $\mu_{\infty}$-almost all $\xi \in X_{\infty}$ are $\mu$-uniformly distributed, we have $\lim _{N \rightarrow \infty} F_{N}(\xi)=0$ for $\mu_{\infty}$-almost all $\xi \in X_{\infty}$.

Let the integer $j \geqq 1$ be given. By Egoroff's theorem ([5] $\S 21 \mathrm{~A}$ ) there exists a subset $\mathfrak{S}_{j}$ of $X_{\infty}$ such that $\mu_{\infty}\left(\mathfrak{S}_{j}\right)>1-\frac{1}{4 j\|f\|}$ and such that the

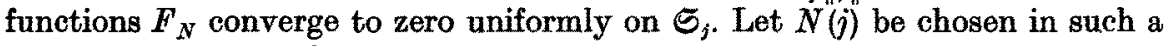
way that $F_{N}(\xi) \leqq \frac{1}{2 j}$ for all $N \geqq N(j)$ and for all $\xi \in \Im_{j}$. We fix an integer $N \geqq N(j)$. By the individual ergodic theorem, applied to the ergodic measure preserving transformation $P^{N}$ in $X_{\infty}$, for all $\xi$ in some subset $\mathfrak{T}(f, j, N) \subset X_{\infty}$ of $\mu_{\infty}$-measure 1 , we have $P^{r N} \xi \in \widetilde{S}_{j}$ for all $r \geqq 0$ except for a subsequence $\left(r_{h}\right)_{h=1}^{\infty}$ of the non-negative integers of asymptotic density smaller than $\frac{1}{4 i|f| !}$. For every such $\xi=\left(x_{n}\right)_{n=1}^{\infty} \in \mathfrak{T}(f, j, N)$ we obtain

$$
\begin{aligned}
& \lim _{R \rightarrow \infty} \sup _{\frac{1}{R}} \sum_{r=0}^{R-1}\left|\frac{1}{N} \sum_{n=r N+1}^{(r+1) N} f\left(x_{n}\right)-\mu(f)\right| \\
& =\limsup _{R \rightarrow \infty} \frac{1}{R} \sum_{r=0}^{R-1} F_{N}\left(P^{r N} \xi\right) \leqq
\end{aligned}
$$

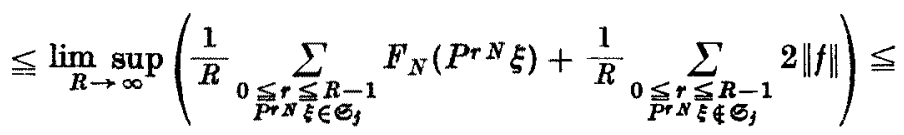

$$
\begin{aligned}
& \leqq \frac{1}{2 j}+\frac{2\|f\|}{4 j\|f\|}=\frac{1}{j} \text {. }
\end{aligned}
$$

For every $\xi \in \bigoplus_{N=N_{(j)}}^{\infty} \mathfrak{T}(f, j, N)$ we therefore have

$$
\limsup _{R \rightarrow \infty} \frac{1}{R} \sum_{r=0}^{R-1}\left|\frac{1}{N} \sum_{n=t+N+1}^{(r+1) N} f\left(x_{n}\right)-\mu(f)\right| \leqq \frac{1}{j} \quad \text { for all } N \geqq N(j)
$$

and for every $\xi \in \mathfrak{T}(f)=\bigcap_{j=1}^{\infty} \bigcap_{N(j)}^{\infty} \mathfrak{T}(f, j, N)$ we have

$$
\lim _{N \rightarrow \infty} \lim _{R \rightarrow \infty} \sup _{\frac{1}{R}} \sum_{r=0}^{R-1}\left|\frac{1}{N} \sum_{n=r+1}^{(r+1) N} f\left(x_{n}\right)-\mu(f)\right|=0 \text {. }
$$


Taking $\mathfrak{T}=\bigcap_{k=1}^{\infty} \mathfrak{T}\left(f_{k}\right)$ we finally have $\mu_{\infty}(\mathfrak{S})=1$ and, by Weyl's criterion, (10) holds for all $f \in C(X)$ and for all $\xi=\left(x_{n}\right)_{n=1}^{\infty} \in \mathcal{T}$.

Corollary 16.1. Suppose that $X$ satisfies the second axiom of countability and that $\mu$ is not a point measure. Then $\mathfrak{W M O} \mathfrak{U P M}_{2}$.

Proof. The statement follows from theorem 16 and corollary 13.1 .

In view of the inclusion $\mathfrak{W} \mathfrak{W}>\mathfrak{A W}_{2}$, theorem 14 furnishes an example of a sequence which is weakly $\mu$-well distributed but not $\mu$-well distributed. Our last two theorems will be concerned with constructions of sequences belonging to the (differences of) classes $\mathfrak{U} \backslash(\mathfrak{A W} \cup \mathfrak{W} \mathfrak{B})$ and $\mathfrak{A W} \backslash\left(\mathfrak{A W} \mathfrak{W}_{1} \cup \mathfrak{W} \mathfrak{W}\right)$ respectively.

Lemma 3. Let $A$ be a closed subset of $X$ with $\mu$-zero boundary and such that $\mu(A)=\alpha>0$. If the sequence $\xi=\left(x_{n}\right)_{n=1}^{\infty}$ is $\mu$-uniformly (resp. $\mu$-well) distributed in $X$, then the subsequence $\eta=\xi \cap A=\left(y_{k}\right)_{k=1}^{\infty}$, consisting of all elements of $\xi$ lying in $A$, is $\frac{1}{\alpha} \mu$-uniformly (resp. $\frac{1}{\alpha} \mu$-well) distributed in $A$, considered as a compact Hausdorff space in the relative topology.

Proof. Since every continuous complex-valued function on $A$ is of the form $f \chi_{A}(f \in C(X))$ by Tietze's extension theorem, we have to show that

$$
\lim _{N \rightarrow \infty}\left|\frac{1}{N} \sum_{k=1}^{N} f \chi_{A}\left(y_{k}\right)-\frac{1}{\alpha} \mu\left(f \chi_{A}\right)\right|=0 \text { for all } f \in C(X),
$$

resp. that

$$
\lim _{N \rightarrow \infty}\left|\frac{1}{N} \sum_{k=m+1}^{m+N} f \chi_{A}\left(y_{k}\right)-\frac{1}{\alpha} \mu\left(f \chi_{A}\right)\right|=0 \text { for all } f \in C(X)
$$

uniformly in $m=0,1,2, \ldots$

Let $f \in C(X)$ be given and suppose first that $\xi$ is $\mu$-uniformly distributed in $X$. For every given integer $N \geqq 1$ we define $N^{\prime}$ to be the smallest integer having the property that $\sum_{n=1}^{N^{\prime}} \chi_{A}\left(x_{n}\right)=N$. Thus, we have $N^{\prime} \geqq N$ and

$$
\lim _{N \rightarrow \infty} \frac{N}{\alpha N^{\prime}}=\frac{1}{\alpha} \cdot \lim _{N^{\prime} \rightarrow \infty} \frac{1}{N^{\prime}} \sum_{n=1}^{N^{\prime}} \chi_{A}\left(x_{n}\right)=1 .
$$

From the identity

$$
\left|\frac{1}{N} \sum_{k=1}^{N} f \chi_{A}\left(y_{k}\right)-\frac{1}{\alpha} \mu\left(f \chi_{A}\right)\right|=\frac{1}{\alpha}\left|\frac{\alpha N^{\prime}}{N} \cdot \frac{1}{N^{\prime}} \sum_{n=1}^{N^{\prime}} f \chi_{A}\left(x_{n}\right)-\mu\left(f \chi_{A}\right)\right|
$$

we then deduce (11).

Suppose next that $\xi$ is $\mu$-well distributed. For every integer $m \geqq 0$ we now $m^{\prime}+1$

define $m^{\prime}$ as the smallest integer having the property that $\sum_{n=1}^{m_{m^{\prime}}+N(m)} \chi_{A}\left(x_{n}\right)=m+1$, and $N(m)$ as the smallest integer having the property that $\sum_{n=m^{\prime}+1}^{m^{\prime}+N(m)} \chi_{A}\left(x_{n}\right)=N$. 
Thus, we have $N(n) \geqq N$. Furthermore, by the same reasoning as above, if $N$ is greater than a fixed integer $N_{0}$, independent of $m$, then $\frac{N}{\alpha N(m)}$ is close to 1 and the difference

$\left|\frac{1}{N} \sum_{k=m+1}^{m+N} f \chi_{A}\left(y_{k}\right)-\frac{1}{\alpha} \mu\left(f \chi_{A}\right)\right|=\frac{1}{\alpha}\left|\frac{\alpha N(m)}{N} \cdot \frac{1}{N(m)} \sum_{n=m^{\prime}+1}^{m^{\prime}+N(m)} f \chi_{A}\left(x_{n}\right)-\mu\left(f \chi_{A}\right)\right|$ is close to 0 , uniformly in $m=0,1,2, \ldots$ This proves the assertion.

Theorem 17. Suppose that $\mu$ is not a point measure and that there exists a $\mu$-uniformly distributed sequence. Then there also exists a sequence $\xi \in X_{\infty}$, constructed explicitely below, which is $\mu$-uniformly distributed but not almost $\mu$-well distributed and not weakly $\mu$-well distributed. Consequently we have $\mathfrak{U} \supsetneq \mathfrak{Q W}$ and $\mathfrak{U} \supsetneq \mathfrak{W} \mathfrak{W}$.

Proof. Let $A$ be a closed subset of $X$ with $\mu$-zero boundary and such that $0<\mu(A)=\alpha<1$ (our hypothesis on $\mu$ implies the existence of such a set $A$ ). Furthermore, let $B$ be the closure of the complement of $A$. Then also $B$ has $\mu$-zero boundary and we have $\mu(f)=\mu\left(f \chi_{A}\right)+\mu\left(f \chi_{B}\right)$ for every $f \in C(X)$. By lemma 3 there exists a sequence $\eta=\left(y_{k}\right)_{k=1}^{\infty}$ which is $\frac{1}{\alpha} \mu$-uniformly distributed in $A$ and a sequence $\zeta=\left(z_{l}\right)_{l=1}^{\infty}$ which is $\frac{1}{1-\alpha} \mu$-uniformly distributed in $B$. We construct the sequence $\xi=\left(x_{n}\right)_{n=1}^{\infty}$ by induction as follows: Let $x_{1}=z_{1}, x_{2}=z_{2}$. If $x_{1}, \ldots, x_{2}\left(\begin{array}{l}h \\ 2\end{array}\right)(h \geqq 2)$ have already been chosen such as to comprise certain initial segments of the sequences $\eta$ and $\zeta$, then let $x_{2}\left(\begin{array}{l}h \\ 2\end{array}\right)+1, \ldots$,

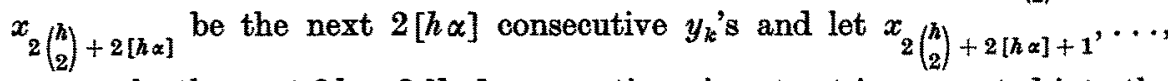
$x_{2\left(\begin{array}{c}h+1 \\ 2\end{array}\right)}$ be the next $2 h-2[h \alpha]$ consecutive $z_{l}$ 's not yet incorporated into the sequence $\xi$ to be constructed.

We first show that the sequence $\xi$ constructed in this way is $\mu$-uniformly distributed in $X$. Let $f \in C(X)$ and $\varepsilon>0$ be given. For a given integer $N \geqq 2$ we determine the integer $h$ in such a way that $2\left(\begin{array}{l}h \\ 2\end{array}\right) \leqq N<2\left(\begin{array}{c}h+1 \\ 2\end{array}\right)$. Furthermore, we define $N_{y}$ (resp. $N_{z}$ ) to be the number of $y_{k}$ 's (resp. $z_{l}$ 's) occurring among the first $2\left(\begin{array}{l}h \\ 2\end{array}\right) x_{n}$ 's. Thus, we have $N_{y z}=N_{y}+N_{x}=2\left(\begin{array}{l}h \\ 2\end{array}\right)$ and

$$
\begin{gathered}
\frac{1}{N} \sum_{n=1}^{N} f\left(x_{n}\right)-\mu(f)= \\
=\frac{N_{y z}}{N}\left\{\frac{N_{y}}{N_{y z}}\left[\frac{1}{N_{y}} \sum_{y} f\left(y_{k}\right)-\frac{1}{\alpha} \mu\left(f \chi_{A}\right)\right]+\left[\frac{N_{y}}{N_{y z}} \cdot \frac{1}{\alpha}-1\right] \mu\left(f \chi_{A}\right)\right\}+ \\
+\frac{N_{y z}}{N}\left\{\frac{N_{z}}{N_{y z}}\left[\frac{1}{N_{z}} \sum_{z} f\left(z_{l}\right)-\frac{1}{1-\alpha} \mu\left(f \chi_{B}\right)\right]+\left[\frac{N_{z}}{N_{y z}} \cdot \frac{1}{1-\alpha}-1\right] \mu\left(f \chi_{B}\right)\right\}+ \\
+\frac{1}{N}\left[\sum_{n=N_{y z}+1}^{N} f\left(x_{n}\right)-\left(N-N_{y z}\right) \mu(f)\right]
\end{gathered}
$$


here $\sum_{y}$ and $\sum_{z}$ denote the sums over the $y_{k}$ 's and $z_{l}$ 's respectively, occurring among the first $N_{y z} x_{n}$ 's, and the third term disappears for $N=N_{y z}$. In view of $\lim _{N \rightarrow \infty} N_{y}=\lim _{N \rightarrow \infty} N_{z}=\infty$ we obtain, for all sufficiently large $N$,

$$
\begin{aligned}
\left|\frac{1}{N} \sum_{n=1}^{N} f\left(x_{n}\right)-\mu(f)\right| & \leqq \frac{N_{y}}{N} \varepsilon+\left|\frac{1}{\alpha} \cdot \frac{N_{y}}{N_{y z}}-1\right|\|f\|+ \\
& +\frac{N_{z}}{N} \varepsilon+\left|\frac{1}{1-\alpha} \cdot \frac{N_{z}}{N_{z z}}-1\right|\|f\|+ \\
& +\frac{4 h}{N}\|f\| .
\end{aligned}
$$

Evidently, for sufficiently large $N$, the last term becomes smaller than $\varepsilon$. Furthermore, we have

$$
\frac{N_{y}}{N_{y z}}=\frac{2[\alpha]+2 \cdot[2 \alpha]+2 \cdot[3 \alpha]+\cdots+2[(h-1) \alpha]}{2 \cdot 1+2 \cdot 2+2 \cdot 3+\cdots+2 \cdot(h-1)} .
$$

Therefore, we conclude $\lim _{N \rightarrow \infty} \frac{N_{\nu}}{N_{y z}}=\alpha$ and, by a similar reasoning, $\lim _{N \rightarrow \infty} \frac{N_{z}}{N_{y z}}$ $=1-\alpha$. Thus, we obtain

$$
\left|\frac{1}{N} \sum_{n=1}^{N} f\left(x_{n}\right)-\mu(f)\right|<4 \varepsilon
$$

for all sufficiently large $N$.

We next show that $\xi$ is not almost $\mu$-well distributed. In order to do this, for every given integer $m \leqq 2$ we estimate the difference $\left|\frac{1}{N} \sum_{n=m+1}^{m+N} \chi_{A}\left(x_{n}\right)-\alpha\right|$ from below for suitably chosen integers $N$. We distinguish four cases, depending on the choice of $m$.

1) $2\left(\begin{array}{l}h \\ 2\end{array}\right) \leqq m<2\left(\begin{array}{l}h \\ 2\end{array}\right)+[h \alpha]$. We choose $N=2\left(\begin{array}{l}h \\ 2\end{array}\right)+2[h \alpha]-m$. Then we have $N>[h \alpha]$ and

$$
\left|\frac{1}{N} \sum_{n=m+1}^{m+N} \chi_{A}\left(x_{n}\right)-\alpha\right|=1-\alpha>0 .
$$

2) $2\left(\begin{array}{l}h \\ 2\end{array}\right)+[h \alpha] \leqq m<2\left(\begin{array}{l}h \\ 2\end{array}\right)+2[h \alpha]$. We choose $N=2\left(\begin{array}{c}h+1 \\ 2\end{array}\right)-m$. Then we have $N>2 h-2[h \alpha]$ and

$$
\frac{1}{N} \sum_{n=m+1}^{m+N} \chi_{A}\left(x_{n}\right) \leqq \max _{1 \leqq j \leqq[h \alpha]} \frac{j}{2 h-2[h \alpha]+j}=\frac{[h \alpha]}{2 h-[h \alpha]} \leqq \frac{\alpha}{2-\alpha}<\alpha .
$$

Therefore, we obtain

$$
\left|\frac{1}{N} \sum_{n=m+1}^{m+N} \chi_{A}\left(x_{n}\right)-\alpha\right| \geqq \alpha-\frac{\alpha}{2-\alpha}=\alpha \cdot \frac{1-\alpha}{2-\alpha}>0 .
$$

3) $2\left(\begin{array}{l}h \\ 2\end{array}\right)+2[h \alpha] \leqq m<2\left(\begin{array}{l}h \\ 2\end{array}\right)+h+[h \alpha]$. We choose $N=2\left(\begin{array}{c}h+1 \\ 2\end{array}\right)-m$. Then we have $N>h-[h \alpha]$ and

$$
\left|\frac{1}{N} \sum_{n=m+1}^{m+N} \chi_{A}\left(x_{n}\right)-\alpha\right|=\alpha>0 \text {. }
$$


4) $2\left(\begin{array}{l}h \\ 2\end{array}\right)+h+[h \alpha] \leqq m<2\left(\begin{array}{c}h+1 \\ 2\end{array}\right)$. We choose $N=2\left(\begin{array}{c}h+1 \\ 2\end{array}\right)+$ $+2[(h+1) \alpha]-m$. Then we have $N>2[(h+1) \alpha]$ and

$$
\frac{1}{N} \sum_{n=m+1}^{m+N} \chi_{A}\left(x_{n}\right) \geq \frac{2[(h+1) \alpha]}{h-[h \alpha]+2[(h+1) \alpha]} .
$$

For large $h$ the right member is close to $\frac{2 \alpha}{1+\alpha}>\alpha$. As a consequence, for large $h$ we obtain

$$
\left|\frac{1}{N} \sum_{n=m+1}^{m+N} \chi_{A}\left(x_{n}\right)-\alpha\right| \geqq \frac{1}{2}\left(\frac{2 \alpha}{1+\alpha}-\alpha\right)=\frac{\alpha}{2} \cdot \frac{1-\alpha}{1+\alpha}>0 .
$$

Choosing now $\varepsilon=\min \left(\alpha \cdot \frac{1-\alpha}{2-\alpha}, \frac{\alpha}{2} \cdot \frac{1-\alpha}{1+\alpha}\right)$, we see that, given any positive integer $N_{0}$, for every integer $m$ which is larger than a certain constant $M$ depending on $N_{0}$ we can find an integer $N_{m} \geqq N_{0}$ such that

$$
\left|\frac{1}{N_{m}} \sum_{n=m+1}^{m+N_{m}} \chi_{A}\left(x_{n}\right)-\alpha\right| \geqq \varepsilon .
$$

It is therefore not possible to select an infinite increasing sequence of integers $m_{k}$ in such a way that the sequences $P^{m_{k}} \xi(k=1,2, \ldots)$ are equi- $\mu$-uniformly distributed. Thus, $\xi$ is not almost $\mu$-well distributed.

Finally we show that the sequence $\xi$ is not weakly $\mu$-well distributed. In fact, we shall prove that, for every fixed integer $N \geqq 1$,

$$
\lim _{R \rightarrow \infty} \frac{1}{R} \sum_{r=0}^{R-1}\left|\frac{1}{N} \sum_{n=r N+1}^{(r+1) N} \chi_{A}\left(x_{n}\right)-\alpha\right|=2 \alpha(1-\alpha) .
$$

To this purpose it will suffice to show the following: Given any integer $h \geqq 2$, let $\left(x_{r N+1}, \ldots, x_{(r+1)}\right)\left(r_{h-1}<r \leqq r_{h}\right)$ be those blocks of $N$ consecutive elements of $\xi$ that have some overlap with the block of $2[h \alpha]$ consecutive elements $y_{k}$ and $2 h-2[h \alpha]$ consecutive elements $z_{l}$, but no overlap with the following block of $2[(h+1) \alpha]$ consecutive elements $y_{k}$ and $2(h+1)-2[(h+1) \alpha]$ consecutive elements $z_{l}$ in the same sequence $\xi$. Then

$$
\lim _{h \rightarrow \infty} \frac{1}{r_{n}-r_{n-1}} \sum_{r=r_{h-1}+1}^{r_{A}}\left|\frac{1}{N} \sum_{n=r+N+1}^{(r+1) N} \chi_{A}\left(x_{n}\right)-\alpha\right|=2 \alpha(1-\alpha) .
$$

(We note that $\frac{2 h}{N}-1 \leqq r_{h}-r_{h-1} \leqq \frac{2 h}{N}+1$. From this follows $\lim _{h \rightarrow \infty}\left(r_{h}-r_{h-1}\right)$ $=\infty$ and

$$
\begin{array}{r}
\lim _{n \rightarrow \infty} \frac{1}{r_{n}-r_{n-1}} \sum_{r=}^{r_{n-1}}\left|\frac{1}{N} \sum_{n=r N+1}^{(r+1) N} \chi_{A}\left(x_{n}\right)-\alpha\right| \\
\left.=\lim _{R \rightarrow \infty} \frac{1}{R} \sum_{r=0}^{R-1}\left|\frac{1}{N} \sum_{n=r N+1}^{(r+1) N} \chi_{A}\left(x_{n}\right)-\alpha\right|\right) .
\end{array}
$$


Indeed, if $h$ is sufficiently large, for approximately $\frac{2[h \alpha]}{N}\left(\right.$ at least $\left.\frac{2[h \alpha]}{N}-2\right)$ values of $r\left(r_{n-1}<r \leqq r_{h}\right)$ the difference $d_{r}=\left|\frac{1}{N} \sum_{n=r N+1}^{(r+1) N} \chi_{A}\left(x_{n}\right)-\alpha\right|$ will have the value $1-\alpha$, while for approximately $\frac{2 h-2[h \alpha]}{N}$ (at least $\frac{2 h-2[h \alpha]}{N}-2$ ) values of $r\left(r_{h-1}<r \leqq r_{h}\right)$ this value will be $\alpha$ and for at most two values of $r, d_{r}$ will be different from $\alpha$ and $1-\alpha$ but bounded above by 2 . Thus we obtain

$$
\begin{aligned}
\lim _{h \rightarrow \infty} & \frac{1}{r_{h}-r_{h-1}} \sum_{r=r_{h-1}+1}^{r_{n}}\left|\frac{1}{N} \sum_{n=r N+1}^{(r+1) N} \chi_{A}\left(x_{n}\right)-\alpha\right| \\
& =\lim _{h \rightarrow \infty} \frac{N}{2 h} \cdot \frac{2[h \alpha](1-\alpha)+(2 h-2[h \alpha]) \alpha}{N}=2 \alpha(1-\alpha) .
\end{aligned}
$$

This completes the proof.

In the mod 1 case, a less abstract example of a sequence enjoying the properties mentioned in theorem 17 (having also served as a model for the construction carried out in the proof) is furnished by the sequence $\left(0,0, \frac{1}{2}\right.$, $\left.0, \frac{1}{3}, \frac{2}{3}, 0, \frac{1}{4}, \frac{2}{4}, \frac{3}{4}, 0, \frac{1}{5}, \ldots\right)$. Sequences of this type have been considered in [16] in connection with the rearrangement of arbitrary sequences, every where dense $\bmod 1$, to $\bmod 1$ uniformly distributed sequences.

Theorem 18. Suppose that $\mu$ is not a point measure and that there exists a $\mu$-well distributed sequence. Then there also exists an almost $\mu$-well but not weakly $\mu$-well distributed sequence $\omega \in X_{\infty}$, constructed explicitely below, with the following property: if $\mathfrak{M}=\left(m_{k}\right)_{k=1}^{\infty}$ is any infinite subsequence of the nonnegative integers such that $\omega$ is almost $\mu$-well distributed $\left(m_{k}\right)_{k=1}^{\infty}$, then $\mathfrak{M}$ has asymptotic density 0 . Consequently we have $\mathfrak{A} \mathfrak{W} \mathfrak{A} \mathfrak{W}_{1}$ and $\mathfrak{W} \mathfrak{D} \mathfrak{D} \mathfrak{W}$.

Proof. As in the proof of theorem 17, let $A$ be a closed subset of $X$ with $\mu$-zero boundary and such that $0<\mu(A)=\alpha<1$, and let $B$ be the closure of the complement of $A$. Let $\xi=\left(x_{k}\right)_{k=1}^{\infty}$ be a sequence which is $\mu$-well distributed in $X$, let $\eta=\left(y_{l}\right)_{l=1}^{\infty}$ be $\frac{1}{\alpha} \mu$-well distributed in $A$ and let $\zeta=\left(z_{m}\right)_{m=1}^{\infty}$ be $\frac{1}{1-\alpha} \mu$-well distributed in $B$ (cf. lemma 3 ). We construct the sequence $\omega=\left(u_{n}\right)_{n=1}^{\infty}$ by induction as follows: Let $u_{1}=x_{1}$. If $u_{1}, \ldots, u_{h^{*}}(h \geqq 1)$ have already been chosen such as to comprise certain initial segments of the sequences $\xi$, $\eta$, and $\zeta$, let $u_{h^{4}+1}, \ldots, u_{h^{4}+6 h^{2}+1}$ be the next $6 h^{2}+1$ consecutive $x_{k}$ 's, and let $u_{h^{4}+6 h^{2}+2}, \ldots, u_{(h+1)^{4}}$ consists of the $2 h^{2}+2$ consecutive blocks of $2 h$ elements each (referred to in the following as " $y z$-blocks"), which in turn consist of the next $2[h \alpha]$ consecutive $y_{l}$ 's and, immediately following, the next $2 h-2[h \alpha]$ consecutive $z_{m}$ 's not yet incorporated into the sequence $\omega$ to be constructed.

We shall first show that the sequence $\omega$ is almost $\mu$-well distributed $\left(h^{4}\right)_{h=1}^{\infty}$ To this purpose, given any $f \in C(X)$ and any $\varepsilon>0$, we choose $N_{1}$ such that, 
uniformly in $h=1,2, \ldots$,

$$
\begin{aligned}
& \left|\frac{1}{N} \sum_{k=h+1}^{h+N} f\left(x_{k}\right)-\mu(f)\right| \leqq \varepsilon \\
& \left|\frac{1}{N} \sum_{l=h+1}^{h+N} f\left(y_{l}\right)-\frac{1}{\alpha} \mu\left(f \chi_{A}\right)\right| \leqq \varepsilon \\
& \left|\frac{1}{N} \sum_{m=h+1}^{h+N} f\left(z_{m}\right)-\frac{1}{1-\alpha} \mu\left(f \chi_{B}\right)\right| \leqq \varepsilon
\end{aligned} \mid \text { for all } N \geqq N_{1} .
$$

Furthermore, we choose the integer $H$ such that $\min (2[H \alpha], 2 H-2[H \alpha]) \geqq N_{1}$ and such that $\max \left(\frac{4 h}{6 h^{2}+1},\left|\frac{[h \alpha]}{h \alpha}-1\right|,\left|\frac{h-[h \alpha]}{h(1-\alpha)}-1\right|\right) \leqq \varepsilon$ for all $h \geqq H$. Let now $h \geqq H$ and $N \geqq N_{1}$ be given. We define $N_{x}$ to be the number of $x_{k}$ 's among the elements $u_{h^{4}+1}, \ldots, u_{h^{4}+N}$. Furthermore, consider the $y z-$ blocks completely covered by these elements and let $N_{v}$ and $N_{z}$ be the number of the $y_{l}$ 's and $z_{m}$ 's contained in all of these $y z$-blocks respectively. We set $N_{y z}=N_{y}+N_{z}$ and $N_{u}=N-N_{x}-N_{y}-N_{z}$. Then we have

$$
\begin{gathered}
\frac{1}{N} \sum_{n=h^{4}+1}^{h^{4}+N} f\left(u_{n}\right)-\mu(f)=\frac{N_{x}}{N}\left[\frac{1}{N_{x}} \sum_{x} f\left(x_{k}\right)-\mu(f)\right]+ \\
+\frac{N_{y z}}{N}\left\{\frac{N_{y}}{N_{y z}}\left[\frac{1}{N_{y}} \sum_{y} f\left(y_{l}\right)-\frac{1}{\alpha} \mu\left(f \chi_{A}\right)\right]+\left[\frac{N_{y}}{\alpha N_{y z}}-1\right] \mu\left(f \chi_{A}\right)\right\}+ \\
+\frac{N_{y z}}{N}\left\{\frac{N_{z}}{N_{y z}}\left[\frac{1}{N_{z}} \sum_{z} f\left(z_{m}\right)-\frac{1}{1-\alpha} \mu\left(f \chi_{B}\right)\right]+\left[\frac{N_{z}}{(1-\alpha) N_{y z}}-1\right] \mu\left(f \chi_{B}\right)\right\}+ \\
+\frac{1}{N}\left[\sum_{u} f\left(u_{n}\right)-N_{u} \mu(f)\right] .
\end{gathered}
$$

Here the sums $\sum_{x}, \sum_{y}, \sum_{z}$ are extended over the corresponding $N_{u}, N_{y}, N_{z}$ elements $x_{k}, y_{l}, z_{m}$ respectively. The second and third member disappear if $N<6 h^{2}+1+2 h$; the fourth member also may disappear and contains a sum $\sum_{u}$ over $N_{u}$ tail elements where $N_{u}$ (if positive) is less than the number of elements in the unfinished $y z$-block. Taking absolute values, because of $N \geqq N_{1}$ and $6 h^{2}+1 \geqq \min (2[h \alpha], 2 h-2[h \alpha]) \geqq N_{1}$ we obtain

$$
\begin{aligned}
\mid \frac{1}{N} \sum_{n=h^{4}+1}^{h^{4}+N} f\left(u_{n}\right) & -\mu(f) \mid \leqq \frac{N_{x}}{N} \varepsilon+ \\
& +\frac{N_{y z}}{N}\left\{\frac{N_{y}}{N_{y z}} \varepsilon+\left|\frac{N_{y}}{\alpha N_{y z}}-1\right|\|f\|\right\}+ \\
& +\frac{N_{y z}}{N}\left\{\frac{N_{z}}{N_{v z}} \varepsilon+\left|\frac{N_{z}}{(1-\alpha) N_{y z}}-1\right|\|f\|\right\}+ \\
& +\frac{2 h}{6 h^{2}+1} \cdot 2\|f\| \leqq \\
& \leqq \varepsilon+\left|\frac{N_{y}}{\alpha N_{y z}}-1\right|\|f\|+\left|\frac{N_{z}}{(1-\alpha) N_{y z}}-1\right|\|f\|+\varepsilon\|f\| .
\end{aligned}
$$


Let the integer $i \geqq 1$ be chosen such that $(h+i-1)^{4}<h^{4}+N \leqq(h+i)^{4}$. We then have, for some integer $j \geqq 0$,

$$
\begin{aligned}
& N_{y z}=2 h\left(2 h^{2}+2\right)+2(h+1)\left(2(h+1)^{2}+2\right)+\cdots+2(h+i-1) \cdot j \\
& N_{y}=2[h \alpha]\left(2 h^{2}+2\right)+2[(h+1) \alpha]\left(2(h+1)^{2}+2\right)+\cdots+2[(h+i-1) \alpha] \cdot j \\
& N_{z}=2(h-[h \alpha])\left(2 h^{2}+2\right)+2(h+1-[(h+1) \alpha])\left(2(h+1)^{2}+2\right)+\cdots+ \\
& \quad+2(h+i-1-[(h+i-1) \alpha]) \cdot j
\end{aligned}
$$

and therefore

$$
\min \left(\frac{[h \alpha]}{h}, \ldots, \frac{[(h+i-1) \alpha]}{h+i-1}\right) \leqq \frac{N_{v}}{N_{y z}} \leqq \max \left(\frac{[h \alpha]}{h}, \ldots, \frac{[(h+i-1) \alpha]}{h+i-1}\right) .
$$

By our ehoice of $h \geqq H$ we conclude $\left|\frac{N_{y}}{\alpha N_{y z}}-1\right| \leqq \varepsilon$ and, analogously, $\frac{N_{x}}{(1-\alpha) N_{y x}}-1 \mid \leqq \varepsilon$. Thus we obtain

$$
\left|\frac{1}{N} \sum_{n=h^{4}+1}^{h^{4}+N} f\left(u_{n}\right)-\mu(f)\right| \leqq \varepsilon+3 \varepsilon\|f\| \text { for all } N \geqq N_{1}
$$

and for all $h \geqq H$. This proves that $\omega$ is almost $\mu$-well distributed $\left(h^{4}\right)_{h=1}^{\infty}$.

Suppose next that the subsequence $\mathfrak{M}=\left(m_{k}\right)_{k=1}^{\infty}$ of the non-negative integers has positive upper asymptotic density. We shall show that the sequences $P^{m_{k}} \omega(k=1,2, \ldots)$ cannot be equi- $\mu$-uniformly distributed. Consider the set of positive integers $\mathfrak{R}=\left\{n: h^{4}-6(h-1)^{2} \leqq n \leqq h^{4}+6 h^{2}\right.$ for some $h=1,2, \ldots\}$. Since $\mathfrak{R}$ has asymptotic density zero, infinitely many $m_{k} \in \mathfrak{M}$ must lie outside of $\mathfrak{R}$ and therefore satisfy the inequality

$$
h^{4}+6 h^{2}+1 \leqq m_{k} \leqq(h+1)^{4}-6 h^{2}-1 \quad(h \geqq 1 \text { an integer })
$$

It follows that the element $u_{m_{k}+1}$ belongs to some $y z$-block of length $2 h$ and, since $m_{k}+4 h<(h+1)^{4}$, the elements $u_{m_{k}+n}(1 \leqq n \leqq 4 h)$ cover at least one complete $y z$-block of length $2 h$. Since this happens for infinitely many $k$, by the same reasoning as in the proof of theorem 17 , the sequences $P^{m_{k}} \omega$ $(k=1,2, \ldots)$ cannot be equi- $\mu$-uniformly distributed.

Finally, since the elements $x_{k}$ form a subsequence of asymptotic density 0 in $\omega$, by the same reasoning as in the proof of theorem 17 we find that

$$
\lim _{R \rightarrow \infty} \frac{1}{R} \sum_{r=0}^{R-1}\left|\frac{1}{N} \sum_{n=r N+1}^{(r+1) N} \chi_{A}\left(u_{n}\right)-\alpha\right|=2 \alpha(1-\alpha) .
$$

for all $N \geqq 1$. Thus, the sequence $\omega$ cannot be weakly $\mu$-well distributed. This completes the proof.

The preceding discussion still leaves some open questions concerning the remaining possible inclusions between the classes $\mathfrak{A W}$ and $\mathfrak{A W}_{1}$ on the one hand and $\mathfrak{W} \mathfrak{W}$ on the other hand. Apart from these we mention one particular question which so far has not been taken up: Suppose that $X$ satisfies the second axiom of countability. Does there always exist a $\mu$-well distributed sequence? In a recent publication, written after completion of the present 
paper, BAAYEN and HEDrLfN [1] showed that the answer is yes. Thus the hypothesis of existence of a $\mu$-well distributed sequence in theorem 14 and theorem 18 (as well as the hypothesis of existence of a $\mu$-uniformly distributed sequence in theorem 17) may be replaced by the second countability axiom.

\section{References}

[1] BAAYEN, P. C., and Z. HEDRLfN: On the existence of well distributed sequences in compact spaces. Proc. Koninkl. Ned. Akad. Wetenschap. Ser. A $68=$ Indag. Math. 27, 221-228 (1965).

[2] vas Dantzic, D.: Zur topologischen Algebra. I. Komplettierungstheorie. Math. Ann. 107, 587-626 (1933).

[3] Eckmans, B.: Otber monothetische Gruppen. Comment. Math. Helv. 16, 249-263 $(1943 / 44)$.

[4] Halmos, P. R., and H. Samelson: On monothetic groups. Proc. Nat. Acad. Sci. U.S.A. 28, 254-258 (1942).

[5] Halmos, P. R.: Measure theory. New York: Van Nostrand 1956.

[6] - Lectures on ergodio theory. Publ. Math. Soc. Japan 3, Tokyo 1958.

[7] Hartman, S., u. C. Ryll-Nardzewski: Zur Theorie der lokalkompakten abelschen Gruppen. Colloq. Math. 4, 157-188 (1957).

[8] Hermagra, G., and A. Paalman-de Miranda: Almost no sequence is well distributed. Proc. Koninkl. Ned. Akad. Wetenschap. Ser. A 67, = Indag. Math. 86, 488-492(1964).

[9] Hewrrr, E., and K. A. Ross: Abstract harmonie analysis I. Berlin-Göttingen-Heidelberg: Springer Verlag 1963.

[10] HLawkA, E.: Zur formalen Theorie der Gleichverteilung in kompakten Gruppen. Rend. Circ. Mat. Palermo, Ser. 2, 4, 33-47 (1955).

[11] - ther einen Satz von vay DER CorPot. Arch. Math. 6, 115-120 (1955).

[12] - Folgen auf kompakten Räumen. Abhandl. math. Seminar hamburg. Univ. 20, 223-241 (1956).

[13] - Erbliche Eigenschaften in der Theorie der Gleichverteilung. Publ. Math. Debrecen 7, 181-186 (1960).

[14] Koksma, J. F.: Diophantische Approximationen. Ergebn. Math. u. Grenzgeb. Bd. 4, Heft 4. Berlin-Göttingen-Heidelberg: Springer-Verlag 1936.

[15] KоRoвov, N. M.: On completely uniform distribution and jointly normal numbers (Russian). Izv. Akad. Nauk S.S.S.R. Ser. Mat. 20, 649-680 (1956).

[16] von NwUManN, J.: GleichmäBig dichte Zahlenfolgen (Hungerian, German summary). Math. Phys. Lapok 32, 32-40 (1925).

[17] Petressen, G. M.: "Almost convergence" and uniformly distributed sequences. Quart. J. Math. Oxford. Ser. 2, 7, 188-191 (1956).

[18] PonTruacn, L. S.: Topologische Gruppen I, II. Leipzig: Teubner-Verlag 1957/58. 